\title{
Stabilized finite element method for the transient Navier- Stokes equations based on a pressure gradient projection
}

\author{
Ramon Codina *, Jordi Blasco \\ Universitat Politècnica de Catalunya, Jordi Girona 1-3, Edifici C1, 08034 Barcelona, Spain
}

Received 1 September 1998

\begin{abstract}
In this paper we present a stabilized finite element formulation for the transient incompressible Navier-Stokes equations. The main idea is to introduce as a new unknown of the problem the projection of the pressure gradient onto the velocity space and to add to the incompresibility equation the difference between the Laplacian of the pressure and the divergence of this new vector field. This leads to a pressure stabilization effect that allows the use of equal interpolation for both velocities and pressures. In the case of the transient equations, we consider the possibility of treating the pressure gradient projection either implicitly or explicity. In the first case, the number of unknowns of the problem is substantially increased with respect to the standard Galerkin formulation. Nevertheless, iterative techniques may be used in order to uncouple the calculation of the pressure gradient projection from the rest of unknowns (velocity and pressure). When this vector field is treated explicitly, the increment of computational cost of the stabilized formulation with respect to the Galerkin method is very low. We provide a stability estimate for the case of the simple backward Euler time integration scheme for both the implicit and the explicit treatment of the pressure gradient projection. (C) 2000 Elsevier Science S.A. All rights reserved.
\end{abstract}

\section{Introduction}

The purpose of this paper is to present a stabilized finite element method for the transient Navier-Stokes equations that allows to use equal velocity-pressure interpolation. This is possible due to a stabilization technique based on the introduction as unknown of the discrete problem of the projection of the pressure gradient onto the finite element space of continuous vector fields. The divergence of the difference between these two vectors (pressure gradient and its projection) is introduced in the continuity equation.

The idea of this stabilization method was originally developed for the stationary Stokes problem in [1] and extended to the non-linear Navier-Stokes problem in [2]. The motivation for the design of this formulation was to inherit the stability properties of some fractional step time integration schemes that use a Poisson equation to compute the pressure (see [3] for further discussion). From the computational point of view, the main drawback of this approach is the introduction of the projection of the pressure gradient as a new unknown of the problem, thus increasing substantially the number of nodal unknowns of the final discrete system. However, iterative strategies may be devised to make the method efficient, with a computational cost similar to that of other stabilization methods.

When the transient Navier-Stokes equations are discretized in time using a finite difference scheme, the projection of the pressure gradient can be treated explicitly, that is to say, evaluated at the previous time step. In this case, the increase of cost of the formulation with respect to the standard Galerkin method is

\footnotetext{
* Corresponding author.

E-mail address: codina@cimne.upc.es (R. Codina).
} 
very low. First, a stabilization matrix must be built up and at the end of each time step the pressure gradient must be projected. This leads to a linear system of equations with a Gramm system matrix which can be solved by a simple Jacobi iteration or approximated by a diagonal system. The number of unknowns is not increased.

In this paper it is shown that the formulation just described is stable. A stability estimate is obtained for both velocities and pressures, without the need to satisfy the standard inf-sup condition on the velocity and pressure finite element spaces encountered when the Galerkin method is used. The stabilization technique is applied to both a monolithic velocity-pressure formulation of the problem using the trapezoidal rule and to an incremental fractional step method.

It has to be remarked that the only purpose of the stabilization technique presented here is to stabilize the pressure, in a similar way to the methods discussed for example in [4] and [5], even though we consider the most general case of transient Navier-Stokes equations. The instabilities due to the convective term when the viscosity is very small are not considered in our formulation. This is reflected by the fact that the stability estimates that we obtain depend on the viscosity. To overcome this, further modifications of the formulation presented herein are required (see for example Refs. [6,7], where formulations accounting both the convective and the pressure instabilities are analyzed).

\section{Problem statement}

Let us consider the transient Navier-Stokes equations for an incompressible fluid. Let $\Omega$ be an open, bounded and polyhedral domain of $\mathbb{R}^{d}$, where $d=2$ or 3 is the number of space dimensions, $\Gamma=\partial \Omega$ its boundary and $[0, T]$ the time interval of analysis. The Navier-Stokes problem consists in finding a velocity $u$
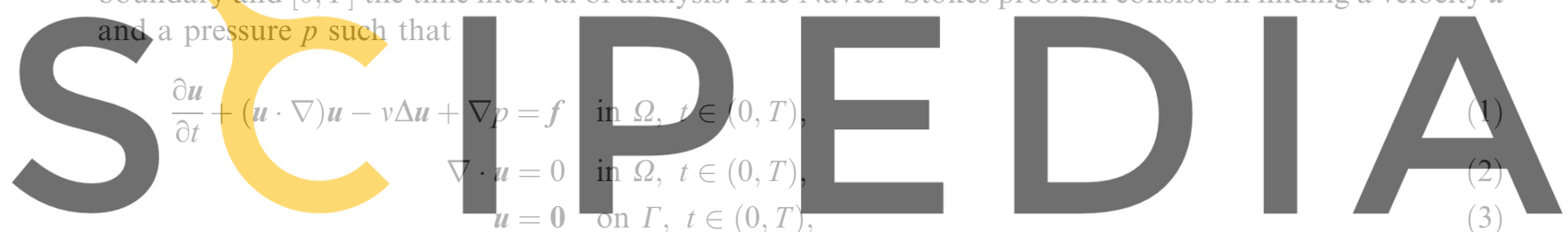

Register for free at https//wwW.scipeđia.com to download the version without the watermark

where $v$ is the kinematic viscosity, $f$ is the force vector and $u^{0}$ is the velocity initial condition. We have considered the homogeneous Dirichiet boundary condition (3) for simplicity.

To write the weak form of problem (1)-(4) we need to introduce some notation. As usual, we denote by $H^{m}(\omega)$ the Sobolev space of $m$ th order in a set $\omega$, consisting of functions whose distributional derivatives of order up to $m$ belong to $L^{2}(\omega)$ and by $H_{0}^{1}(\omega)$ the subspace of $H^{1}(\omega)$ of functions with zero trace on $\Gamma$. A bold character is used for the vector counterpart of these spaces. The $L^{2}$ scalar product is denoted by $(\cdot, \cdot)_{\omega}$, and the $H^{m}$ norm by $\|\cdot\|_{m, \omega}$. The subscript $m$ is omitted when $m=0$ and so is $\omega$ when it is $\Omega$.

Let us now consider the spaces $\boldsymbol{V}_{\mathrm{st}}=\boldsymbol{H}_{0}^{1}(\Omega)^{d}$ and $Q_{\mathrm{st}}=\left\{q \in L^{2}(\Omega) \mid \int_{\Omega} q=0\right\}$ and the forms

$$
\begin{aligned}
a(\boldsymbol{u}, \boldsymbol{v}) & :=v(\nabla \boldsymbol{u}, \nabla \boldsymbol{v}), \\
b(q, \boldsymbol{v}) & :=(q, \nabla \cdot \boldsymbol{v}), \\
c(\boldsymbol{u}, \boldsymbol{v}, \boldsymbol{w}) & :=((\boldsymbol{u} \cdot \nabla) \boldsymbol{v}, \boldsymbol{w})+\frac{1}{2}((\nabla \cdot \boldsymbol{u}) \boldsymbol{v}, \boldsymbol{w}),
\end{aligned}
$$

with $\boldsymbol{u}, \boldsymbol{v}, \boldsymbol{w} \in \boldsymbol{V}_{\text {st }}$ and $q \in Q_{\mathrm{st}}$. All these forms are continuous and $c$ is skew-symmetric in its last two arguments. If $\nabla \cdot \boldsymbol{u}=0$ it coincides with the standard trilinear form coming from the convective term of (1). However, for the finite element formulation that we shall consider the discrete velocity field will not be weakly divergence free and the use of the trilinear form given by (5) simplifies the analysis.

The norms of $a$ and $c$ are denoted by $N_{a}$ and $N_{c}$, respectively, the former being proportional to the viscosity $v$. Moreover, $a$ is coercive as a consequence of the Poincaré-Friedrics inequality, and $b$ satisfies the inf-sup or Babuška-Brezzi condition for the spaces $\boldsymbol{V}_{\text {st }}$ and $Q_{\text {st }}$. 
If $\langle\cdot, \cdot\rangle$ denotes the duality pairing between $\boldsymbol{V}_{\text {st }}$ and its topological dual space $\boldsymbol{V}_{\text {st }}^{\prime}$, the weak form of problem (1)-(4) consists in finding $(\boldsymbol{u}, p) \in \boldsymbol{V} \times Q$, with $\mathbf{V}=\boldsymbol{L}^{2}\left(0, T ; \boldsymbol{V}_{\text {st }}\right)$ and $Q=L^{2}\left(0, T ; Q_{\text {st }}\right)$, such that

$$
\begin{aligned}
\left(\frac{\partial \boldsymbol{u}}{\partial t}, \boldsymbol{v}\right)+c(\boldsymbol{u}, \boldsymbol{u}, \boldsymbol{v})+a(\boldsymbol{u}, \boldsymbol{v})-b(p, \boldsymbol{v}) & =\langle\boldsymbol{f}, \boldsymbol{v}\rangle \quad \forall \boldsymbol{v} \in \boldsymbol{V}_{\mathrm{st}}, \\
b(q, \boldsymbol{u}) & =0 \quad \forall q \in Q_{\mathrm{st}},
\end{aligned}
$$

and satisfying the initial condition in a weak sense. These equations hold weakly in $L^{2}(0, T)$.

If instead of having $\boldsymbol{f} \in \boldsymbol{L}^{2}\left(0, T ; \boldsymbol{H}^{-1}(\Omega)\right)$ we require $\boldsymbol{f} \in \boldsymbol{L}^{2}\left(0, T ; \boldsymbol{L}^{2}(\Omega)\right)$ and $\Gamma$ is sufficiently smooth, it is known that the solution of problem (6) and (7) verifies $\boldsymbol{u} \in \boldsymbol{L}^{2}\left(0, T ; \boldsymbol{V}_{\mathrm{st}} \cap \boldsymbol{H}^{2}(\Omega)\right)$ and $p \in L^{2}(0, T$; $Q_{\text {st }} \cap H^{1}(\Omega)$ ), that is, the regularity of the solution increases (see, e.g., [8]). Also, the duality $\langle\boldsymbol{f}, \boldsymbol{v}\rangle$ in (7) can be replaced by $(\boldsymbol{f}, \boldsymbol{v})$. The finite element approximation proposed in this paper will be based on the fact that $p \in L^{2}\left(0, T ; H^{1}(\Omega)\right)$. We say that problem (1)-(4) is $p$-regular if $p \in L^{2}\left(0, T ; Q_{\mathrm{st}} \cap H^{1}(\Omega)\right)$ whenever $\boldsymbol{f} \in \boldsymbol{L}^{2}\left(0, T ; \boldsymbol{L}^{2}(\Omega)\right)$.

\section{Stabilization of the stationary problem}

Let us consider now the stationary version of problem (1)-(3) and (5) that is, dropping the time dependence in both the velocity and the pressure. In this section we present the stabilized method we have developed for it and collect the most important results we have obtained. In Section 4 we present the extension of this stabilization method to the transient case.

Let $\mathscr{T}_{h}$ denote a finite element partition of the domain $\Omega$ of diameter $h$, from which we construct the finite element spaces $Q_{h}, V_{h}$ and $\boldsymbol{V}_{h, 0}$, approximations to $Q_{\text {st }}, H^{1}(\Omega)^{d}$ and $\boldsymbol{V}_{\text {st }}$, respectively. The former is
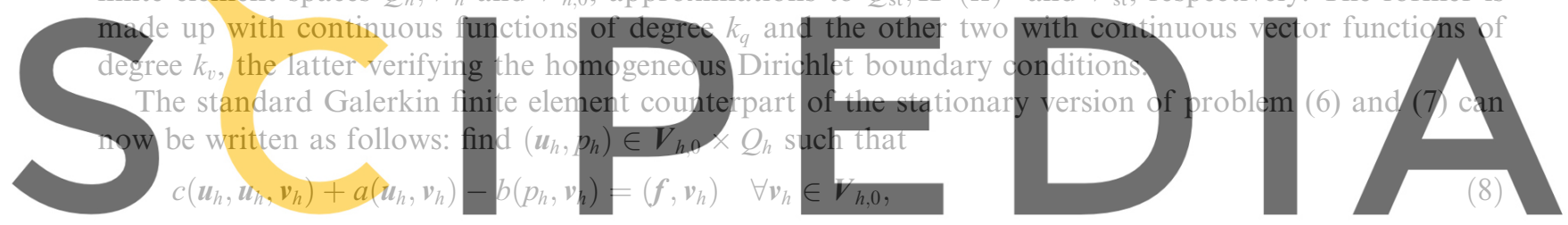

Register for free at https $/ /$ WWw.scipedia.com to download the version without the watermark It is well known that if this formulation is used the velocity and pressure finite element spaces have to satisfy the discrete analogue of the inf-sup condition. In our case this happens if $k_{v}=k_{q}+1$ in the case $d=2$, i.e., for 2D Taylor-Hood type elements $[9,10]$. This condition is not necessary using the method described next.

Let $\alpha_{K}, K \in \mathscr{T}_{h}$, be a family of mesh parameters depending on the element sizes $h_{K}$. The modification of problem (8) and (9) that we propose is: find $\left(\boldsymbol{u}_{h}, p_{h}, \boldsymbol{\xi}_{h}\right) \in \boldsymbol{V}_{h, 0} \times Q_{h} \times \boldsymbol{V}_{h}$ such that

$$
\begin{aligned}
& c\left(\boldsymbol{u}_{h}, \boldsymbol{u}_{h}, \boldsymbol{v}_{h}\right)+a\left(\boldsymbol{u}_{h}, \boldsymbol{v}_{h}\right)-b\left(p_{h}, \boldsymbol{v}_{h}\right)=\left(\boldsymbol{f}, \boldsymbol{v}_{h}\right), \\
& \sum_{K} \alpha_{K}^{2}\left(\nabla p_{h}, \nabla q_{h}\right)_{K}-\sum_{K} \alpha_{K}\left(\boldsymbol{\xi}_{h}, \nabla q_{h}\right)_{K}+b\left(q_{h}, \boldsymbol{u}_{h}\right)=0, \\
& -\sum_{K} \alpha_{K}\left(\nabla p_{h}, \boldsymbol{\eta}_{h}\right)_{K}+\left(\boldsymbol{\xi}_{h}, \boldsymbol{\eta}_{h}\right)=0
\end{aligned}
$$

for all $\left(\boldsymbol{v}_{h}, q_{h}, \boldsymbol{\eta}_{h}\right) \in \boldsymbol{V}_{h, 0} \times Q_{h} \times \boldsymbol{V}_{h}$.

For a function $q \in \boldsymbol{H}^{1}(\Omega)$ let us define $\nabla^{h} q$ by

$$
\left.\nabla^{h} q\right|_{K}=\left.\alpha_{K} \nabla q\right|_{K}, \quad K \in \mathscr{T}_{h} .
$$

From (12) it is seen that $\xi_{h}$ is precisely the projection of $\nabla^{h} p_{h}$ onto $\boldsymbol{V}_{h}$. We shall call it simply the pressure gradient projection. Our stability and convergence analysis is strongly based on a decomposition of the pressure gradient that we describe next. Let $\nabla^{h} Q_{h}$ denote the space of vector functions in $\boldsymbol{L}^{2}(\Omega)$ which are of the form $\nabla^{h} q_{h}$, with $q_{h} \in Q_{h}$ and consider the vector space

$$
\boldsymbol{E}_{h}:=\boldsymbol{V}_{h}+\nabla^{h} Q_{h}=\boldsymbol{E}_{h, 1} \oplus \boldsymbol{E}_{h, 2} \oplus \boldsymbol{E}_{h, 3},
$$


where $\boldsymbol{E}_{h, i}, i=1,2,3$, are three mutually $L^{2}$-orthogonal subspaces defined as

$$
\boldsymbol{E}_{h, 1}:=\boldsymbol{V}_{h, 0}, \quad \boldsymbol{E}_{h, 2}:=\boldsymbol{V}_{h, 0}^{\perp} \cap \boldsymbol{V}_{h}, \quad \boldsymbol{E}_{h, 3}:=\boldsymbol{V}_{h}^{\perp} \cap \boldsymbol{E}_{h} .
$$

Let us denote by $P_{h, i}$ the orthogonal projection from $\boldsymbol{E}_{h}$ to $\boldsymbol{E}_{h, i}$, and $P_{h, i j}:=P_{h, i}+P_{h, j}, i, j=1,2,3$. Also, we denote $\boldsymbol{E}_{h, i j}:=\boldsymbol{E}_{h, i} \oplus \boldsymbol{E}_{h, j}$. In order to prove that the pressure gradient in the discrete problem is stable, we shall bound independently the three terms in the decomposition

$$
\nabla^{h} p_{h}=P_{h, 1}\left(\nabla^{h} p_{h}\right)+P_{h, 2}\left(\nabla^{h} p_{h}\right)+P_{h, 3}\left(\nabla^{h} p_{h}\right) .
$$

The bound for the first component of the pressure gradient decomposition in (14) can be obtained from the momentum Eq. (10), whereas the third component can be bounded only assuming that $\alpha_{K}$ verifies condition (16) stated below. Thus, the stability provided by the method in comparison with the standard problem (8) and (9) is precisely in the control over the term $P_{h, 3}\left(\nabla^{h} p_{h}\right)$, that is, the component of the pressure gradient orthogonal to the space of continuous vector fields $V_{h}$.

The second component in (14) deserves special attention. It depends on the properties of the finite element spaces and not on the problem actually solved. We assume that there is a positive constant $K_{2}^{\prime}$ such that

\section{$\left\|\nabla^{h} q_{h}\right\| \leqslant K_{2}^{\prime}\left\|P_{h, 13}\left(\nabla^{h} q_{h}\right)\right\| \quad \forall q_{h} \in Q_{h}$, \\ which means that $\left\|P_{h, 2}\left(\nabla^{h} q_{h}\right)\right\|$ can be bounded by $\left\|P_{h, 13}\left(\nabla^{h} q_{h}\right)\right\|$. This condition is equivalent to the exis-} tence of a constant $K_{2}=1 / K_{2}^{\prime}>0$ such that

$\inf _{q_{h} \in Q_{h}} \sup _{v_{h} \in E_{h, 13}} \frac{\left(\nabla^{h} q_{h}, v_{h}\right)}{\left\|\nabla q_{h}\right\|\left\|v_{h}\right\|} \geqslant K_{2}$

$$
\frac{N_{c \mid f} \|_{-1}}{K_{a}^{2}}<1,
$$

where $K_{a}$ is the coercivity constant of the bilinear form $a$.

In order to prove stability we need to introduce a further assumption, now on the behavior of the numerical parameters $\alpha_{K}$. There exist constants $\alpha_{0}$ and $\alpha_{1}$, independent of $h_{K}$, such that

$$
\alpha_{0} h_{K} \leqslant \alpha_{K} \leqslant \alpha_{1} h_{K} \quad \forall K \in \mathscr{T}_{h} .
$$

Under this assumption we have:

Theorem 1. Suppose that the uniqueness condition holds and that the family of finite element partitions $\left\{\mathscr{T}_{h}\right\}_{h>0}$ is non-degenerate and such that (15) holds. If $\alpha_{K}$ satisfies (16) then, for $h$ small enough, there exists a unique solution to the discrete finite element problem (10) and (12) that verifies the stability estimate

$$
\left\|\boldsymbol{u}_{h}\right\|_{1}+\left\|\nabla^{h} p_{h}\right\|+\left\|\boldsymbol{\xi}_{h}\right\| \leqslant C\|\boldsymbol{f}\|,
$$

for a constant $C$ independent of $h$.

Let us give now a convergence result for the solution of the discrete problem to the solution of the continuous Navier-Stokes problem in the norm in which stability has been proven, that is, in the $\boldsymbol{H}^{1}$ norm for the velocity and the $L^{2}$ norm of the mesh dependent pressure gradient defined in (13) and its projection 
onto $\boldsymbol{V}_{h}$. This is what we call 'natural' norm of the finite element method presented. Next, $L^{2}$ error estimates can be obtained using classical duality arguments, similar to those using in [5] for the GLS method.

To prove convergence we need to explicitly assume that the problem is $p$-regular. Furthermore, we also need a technical condition on the family of finite element partitions discussed in [2]. Under all these assumptions we can prove [2].

Theorem 2. Suppose that the Navier-Stokes problem is p-regular, the uniqueness condition holds and that the family of finite element partitions $\left\{\mathscr{T}_{h}\right\}_{h>0}$ is non-degenerate and such that (15) holds. Assume also that the parameters $\alpha_{K}$ satisfy (16). Then, for $h$ small enough, the solution of the discrete finite element problem satisfies the error estimate

$$
\left\|\boldsymbol{u}-\boldsymbol{u}_{h}\right\|_{1}+\left\|\nabla^{h} p-\nabla^{h} p_{h}\right\|+\left\|\nabla^{h} p-\xi_{h}\right\| \leqslant C E(h),
$$

where

$$
\begin{aligned}
E(h):= & \inf _{\boldsymbol{v}_{h} \in \boldsymbol{V}_{h, 0}}\left\|\boldsymbol{u}-\boldsymbol{v}_{h}\right\|_{1}+\inf _{\boldsymbol{v}_{h} \in \boldsymbol{V}_{h, 0}} \sum_{K} \frac{1}{h_{K}}\left\|\boldsymbol{u}-\boldsymbol{v}_{h}\right\|_{0, K} \\
& +\inf _{q_{h} \in Q_{h}}\left\|p-q_{h}\right\|+\inf _{q_{h} \in Q_{h}}\left\|\nabla^{h} p-\nabla^{h} q_{h}\right\|+\inf _{\eta_{h} \in V_{h}}\left\|\nabla^{h} p-\eta_{h}\right\|
\end{aligned}
$$

and $\boldsymbol{u}$ and $p$ are the solution of the continuous problem.

Clearly, estimate (17) is optimal. From the standard approximation properties of the finite element spaces it follows that if $\boldsymbol{u} \in \boldsymbol{H}^{r}(\Omega) \cap \boldsymbol{V}, r \geqslant 1$, and $p \in H^{s}(\Omega) \cap Q, s \geqslant 1$, then the error function $E(h)$ in (18) behaves like $h^{k}$, with $k=\min \left\{r-1, s, k_{v}, k_{q}+1\right\}$.

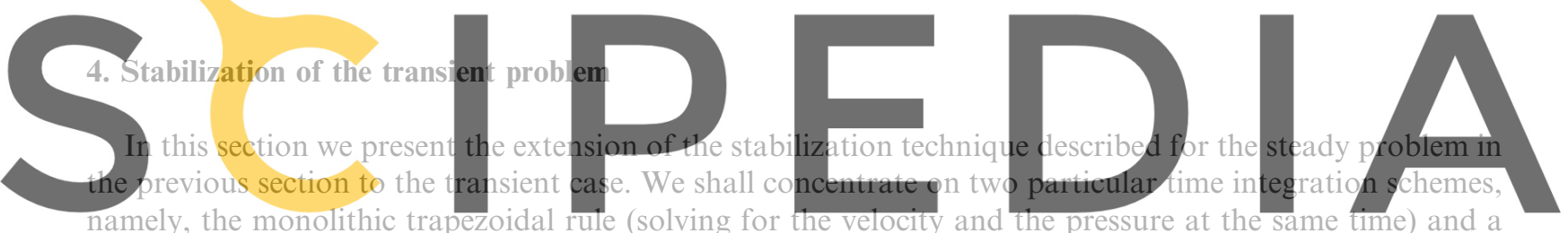

namely, the monolithic trapezoidal rule (solving for the velocity and the pressure at the same time) and a particular fractional step method that allows to compute the different variables by solving uncoupled

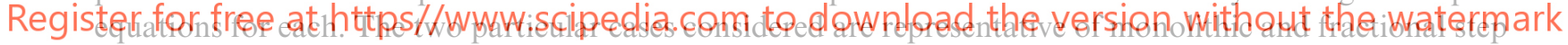

methods and the extension of the stabilization method to other time integration schemes of these types is straightforward.

\subsection{Monolithic schemes}

Let us consider first the trapezoidal rule. The discretization of (6) and (7) in this case consists in solving the following problem: from known $\boldsymbol{u}^{n}$ and $p^{n}$, find $\boldsymbol{u}^{n+1} \in \boldsymbol{V}_{\text {st }}$ and $p^{n+1} \in Q_{\text {st }}$ such that

$$
\begin{aligned}
& \left(\frac{\delta \boldsymbol{u}^{n}}{\delta t}, \boldsymbol{v}\right)+c\left(\boldsymbol{u}^{n+\theta}, \boldsymbol{u}^{n+\theta}, \boldsymbol{v}\right)+a\left(\boldsymbol{u}^{n+\theta}, \boldsymbol{v}\right)-b\left(p^{n+1}, \boldsymbol{v}\right)=\left\langle\boldsymbol{f}^{n+1}, \boldsymbol{v}\right\rangle \quad \forall \boldsymbol{v} \in \boldsymbol{V}_{\mathrm{st}}, \\
& b\left(q, \boldsymbol{u}^{n+1}\right)=0 \quad \forall q \in Q_{\mathrm{st}}
\end{aligned}
$$

where $\delta t$ is the time step size, superscripts refer to the time step level, $\theta \in[0,1]$ and we use the notation

$$
\boldsymbol{u}^{n+\theta}:=\theta \boldsymbol{u}^{n+1}+(1-\theta) \boldsymbol{u}^{n} \text { and } \delta \boldsymbol{u}^{n}:=\boldsymbol{u}^{n+1}-\boldsymbol{u}^{n} .
$$

The force term in (19) and below has to be understood as the time average of the force in the interval $\left[t^{n}, t^{n+1}\right]$, even though we use a superscript $n+1$ to characterize it. The pressure value computed here has been identified as the pressure evaluated at $t^{n+1}$, although this is irrelevant for the velocity approximation. The problem can be written in terms of $\boldsymbol{u}^{n+\theta}$ by expressing $\boldsymbol{u}^{n+1}$ in terms of it. Also, instead of using $c\left(\boldsymbol{u}^{n+\theta}, \boldsymbol{u}^{n+\theta}, \boldsymbol{v}\right)$ for the convective term it is also possible to use a linearized form of it with the same order of approximation. 
The Galerkin finite element approximation of problem (19) and (20) is standard. As for the stationary problem, the finite element spaces used to interpolate the velocity and the pressure need to satisfy the discrete inf-sup condition (see [12] for a complete discussion and analysis of this problem when $\theta=1 / 2$ ). On the other hand, it is possible to avoid the need to satisfy this condition by extending the stabilization technique of the previous section to the transient case. This extension is straightforward. Using the same notation as before, the transient analogue of problem (10) and (12) is to find finite element approximations $\left(\boldsymbol{u}_{h}^{n+\theta}, p_{h}^{n+1}\right)$ to $\left(\boldsymbol{u}^{n+\theta}, p^{n+1}\right)$ and also $\xi_{h}^{n+1}$ such that

$$
\begin{aligned}
& \left(\frac{\delta \boldsymbol{u}_{h}^{n}}{\delta t}, \boldsymbol{v}_{h}\right)+c\left(\boldsymbol{u}_{h}^{n+\theta}, \boldsymbol{u}_{h}^{n+\theta}, \boldsymbol{v}_{h}\right)+a\left(\boldsymbol{u}_{h}^{n+\theta}, \boldsymbol{v}_{h}\right)-b\left(p_{h}^{n+1}, \boldsymbol{v}_{h}\right)=\left(\boldsymbol{f}^{n+1}, \boldsymbol{v}_{h}\right), \\
& \sum_{K} \alpha_{K}^{2}\left(\nabla p_{h}^{n+1}, \nabla q_{h}\right)_{K}-\sum_{K} \alpha_{K}\left(\boldsymbol{\xi}_{h}^{n+\beta}, \nabla q_{h}\right)_{K}+b\left(q_{h}, \boldsymbol{u}_{h}^{n+1}\right)=0, \\
& -\sum_{K} \alpha_{K}\left(\nabla p_{h}^{n+1}, \boldsymbol{\eta}_{h}\right)_{K}+\left(\boldsymbol{\xi}_{h}^{n+1}, \boldsymbol{\eta}_{h}\right)=0
\end{aligned}
$$

for all $\left(v_{h}, q_{h}, \eta_{h}\right) \in \mathbb{V}_{h, 0} \times Q_{h} \times \mathbb{V}_{h}$.

In this equations we have introduced the parameter $\beta$, whose values of interest are $\beta=0$ and $\beta=1$. In the first case, the pressure gradient projection is treated explicitly, whereas in the second it is treated implicitly. We shall see in the next section that this is possible, in the sense that if the parameters $\alpha_{K}$ are properly chosen the resulting time integration scheme is stable.

The choice of $\beta$ is especially relevant from the computational point of view. To discuss this, it is convenient to introduce the matrix form of the algebraic system resulting from problem (21)-(23), which is
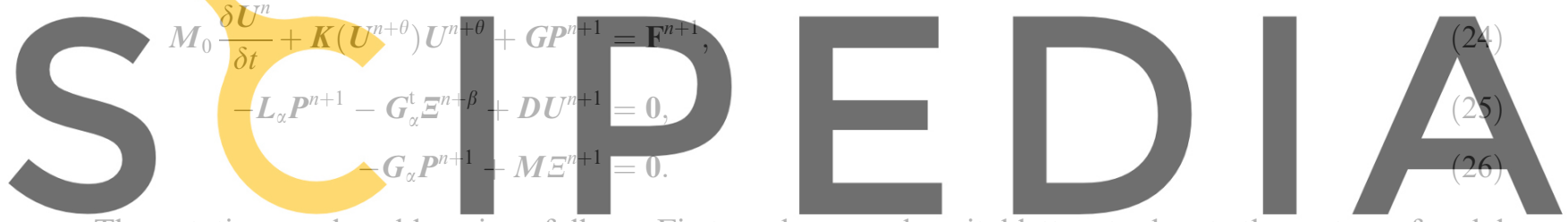

The notation employed here is as follows. First, we have used capital letters to denote the vectors of nodal

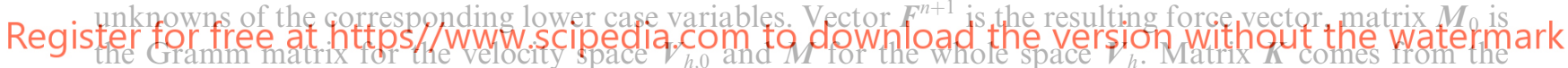

discretization of both the viscous and convective terms and matrices $G, D$ and $L$ come from the approximation of the gradient, the divergence and the Lapiacian operators, respectively. When these matrices are modified by the appearence of the $\alpha_{K}$ parameters in the integrals we have introduced a subscript $\alpha$.

Clearly, when $\beta=0$ Eqs. (24) and (25) can be solved independently of (26), which can be solved for $\boldsymbol{\Xi}^{n+1}$ once the pressure $\boldsymbol{P}^{n+1}$ is known. This makes the stabilized method only marginally more expensive than the standard Galerkin approach. The stability when $\beta=0$ will be discussed in the next section.

From (26) it is found that the nodal unknowns of the pressure gradient projection are

$$
\boldsymbol{\Xi}^{n+\beta}=\boldsymbol{M}^{-1} \boldsymbol{G}_{\alpha} \boldsymbol{P}^{n+\beta} \text {. }
$$

The inversion of $\boldsymbol{M}$ is trivial if it approximated by the standard lumped mass matrix. Otherwise, a linear system needs to be solved, although this can be done efficiently by a sort of iterative techniques.

Inserting the expression for $\boldsymbol{\Xi}^{n+\beta}$ in (25) yields

$$
-\boldsymbol{L}_{\alpha} \boldsymbol{P}^{n+1}-\boldsymbol{G}_{\alpha}^{\mathrm{t}} \boldsymbol{M}^{-1} \boldsymbol{G}_{\alpha} \boldsymbol{P}^{n+\beta}+\boldsymbol{D} \boldsymbol{U}^{n+1}=\mathbf{0} .
$$

This is the modified discrete continuity equation solved by the stabilized method instead of the equation $\boldsymbol{D} \boldsymbol{U}^{n+1}=\mathbf{0}$. For $\beta=1$ it can be written as

$$
\boldsymbol{B}_{\alpha} \boldsymbol{P}^{n+1}+\boldsymbol{D} \boldsymbol{U}^{n+1}=\mathbf{0},
$$

where we have introduced the matrix

$$
\boldsymbol{B}_{\alpha}:=-\boldsymbol{L}_{\alpha}-\boldsymbol{G}_{\alpha}^{\mathrm{t}} \boldsymbol{M}^{-1} \boldsymbol{G}_{\alpha} .
$$


It can be proved that this matrix is positive-semidefinite [3], thus explaining from a purely algebraic point of view why the stability of the pressure is increased by the present stabilization technique.

\subsection{Fractional step schemes}

\subsubsection{Matrix form}

Let us discuss now the application of this stabilization method when a fractional step scheme is used for the time integration. For that, we consider a purely algebraic presentation of these types of methods. Let us split (24) into the following two equations:

$$
\begin{aligned}
& \boldsymbol{M}_{0} \frac{1}{\delta t}\left(\hat{\boldsymbol{U}}^{n+1}-\boldsymbol{U}^{n}\right)+\boldsymbol{K}\left(\boldsymbol{U}^{n+\theta}\right) \boldsymbol{U}^{n+\theta}+\gamma \boldsymbol{G} \boldsymbol{P}^{n}=\boldsymbol{f}^{n+1}, \\
& \boldsymbol{M}_{0} \frac{1}{\delta t}\left(\boldsymbol{U}^{n+1}-\hat{\boldsymbol{U}}^{n+1}\right)+\boldsymbol{G}\left(\boldsymbol{P}^{n+1}-\gamma \boldsymbol{P}^{n}\right)=\mathbf{0},
\end{aligned}
$$

where $\hat{\boldsymbol{U}}^{n+1}$ is an auxiliary vector of unknowns and $\gamma=0$ or 1 . If we write $\hat{\boldsymbol{U}}^{n+\theta}=\theta \hat{\boldsymbol{U}}^{n+1}+(1-\theta) \boldsymbol{U}^{n}$, Eq. (27) can be solved independently of (28) if the approximation

$$
K\left(\hat{U}^{n+\theta}\right) \hat{U}^{n+\theta} \approx K\left(U^{n+\theta}\right) U^{n+\theta}
$$

is used. This is the source of splitting error. Since from (28) it follows that

$$
\boldsymbol{U}^{n+1}=\hat{\boldsymbol{U}}^{n+1}-\delta t \boldsymbol{M}_{0}^{-1} \boldsymbol{G}\left(\boldsymbol{P}^{n+1}-\gamma \boldsymbol{P}^{n}\right)
$$

it is clear that the approximation (29) is formally of order $\mathrm{O}(\delta t)$ if $\gamma=0$ and of order $\mathrm{O}\left(\delta t^{2}\right)$ if $\gamma=1$.
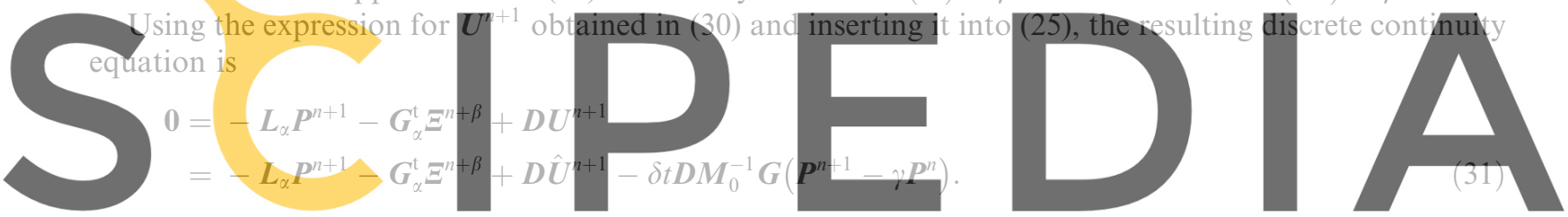

A further approximation is now required in order to make the final discrete problem easier to solve. It

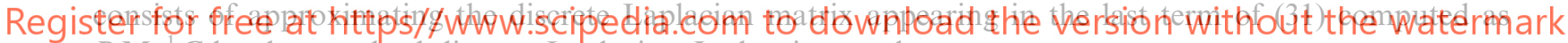

$D M_{0}^{-1} G$ by the standard discrete Laplacian $L$, that is, to take

$$
\boldsymbol{D} \boldsymbol{M}_{0}^{-1} \boldsymbol{G}\left(\boldsymbol{P}^{n+1}-\gamma \boldsymbol{P}^{n}\right) \approx \boldsymbol{L}\left(\boldsymbol{P}^{n+1}-\gamma \boldsymbol{P}^{n}\right) .
$$

Using this, together with the splitting (27) and (28) of (24) and the approximation (29), the final algebraic system of equations to be solved at each time step is

$$
\begin{aligned}
& \boldsymbol{M}_{0} \frac{1}{\delta t}\left(\hat{\boldsymbol{U}}^{n+1}-\boldsymbol{U}^{n}\right)+\boldsymbol{K}\left(\hat{\boldsymbol{U}}^{n+\theta}\right) \hat{\boldsymbol{U}}^{n+\theta}+\gamma \boldsymbol{G P}^{n}=\mathbf{F}^{n+1}, \\
& \delta t \boldsymbol{L}\left(\boldsymbol{P}^{n+1}-\gamma \boldsymbol{P}^{n}\right)=\boldsymbol{D} \hat{\boldsymbol{U}}^{n+1}-\boldsymbol{L}_{\alpha} \boldsymbol{P}^{n+1}-\boldsymbol{G}_{\alpha}^{\mathrm{t}} \boldsymbol{\Xi}^{n+\beta}, \\
& -\boldsymbol{G}_{\alpha} \boldsymbol{P}^{n+1}+\boldsymbol{M} \Xi^{n+1}=\mathbf{0}, \\
& \boldsymbol{M}_{0} \frac{1}{\delta t}\left(\boldsymbol{U}^{n+1}-\hat{\boldsymbol{U}}^{n+1}\right)+\boldsymbol{G}\left(\boldsymbol{P}^{n+1}-\gamma \boldsymbol{P}^{n}\right)=\mathbf{0} .
\end{aligned}
$$

This equations are very attractive from the implementation point of view because they are uncoupled. Eq. (33) can be solved for $\hat{\boldsymbol{U}}^{n+1}$, and then the pressure can be computed directly from (34) if $\beta=0$ or coupling this equation together with (35) if $\beta=1$. Finally, (36) can be solved for $\boldsymbol{U}^{n+1}$.

\subsubsection{Motivation from a continuous problem}

To motivate the method given by (33)-(36) let us assume for a moment that the parameters $\alpha_{K}$ are constant and equal to a value $\alpha$. If we take the trapezoidal rule as given by (21)-(23) as the starting point for 
the design of the fractional step method, it is seen that the continuous equations that are being approximated are

$$
\begin{aligned}
\frac{\partial \boldsymbol{u}}{\partial t}+(\boldsymbol{u} \cdot \nabla) \boldsymbol{u}-v \Delta \boldsymbol{u}+\nabla p & =\boldsymbol{f}, \\
-\alpha^{2} \Delta p+\alpha \nabla \cdot \boldsymbol{\xi}+\nabla \cdot \boldsymbol{u} & =0, \\
-\alpha \nabla p+\boldsymbol{\xi} & =\mathbf{0},
\end{aligned}
$$

with the appropriate boundary conditions. Let us introduce the abbreviation

$$
N(\boldsymbol{u}):=(\boldsymbol{u} \cdot \nabla) \boldsymbol{u}-v \Delta \boldsymbol{u} .
$$

To discretize problem (37)-(39) let us consider the simplest fractional step scheme [13,14], which consists of solving the problem

$$
\begin{aligned}
& \frac{1}{\delta t}\left(\hat{\boldsymbol{u}}^{n+1}-\boldsymbol{u}^{n}\right)+N\left(\hat{\boldsymbol{u}}^{n+1}\right)+\gamma \nabla p^{n}=\boldsymbol{f}^{n+1}, \\
& \frac{1}{\delta t}\left(u^{n+1}-\hat{u}^{n+1}\right)+\nabla\left(p^{n+1}-\gamma p^{n}\right)=0, \\
& -\alpha^{2} \Delta p^{n+1}+\alpha \nabla \cdot \xi^{n+\beta}+\nabla \cdot u^{n+1}=0, \\
& -\alpha \nabla p^{n+1}+\xi^{n+1}=0 .
\end{aligned}
$$

In this equations, $\hat{\boldsymbol{u}}^{n+1}$ is an intermediate velocity which is computed from a pressure guess and for which the continuity equation is not enforced. If Eqs. (40) and (41) are added up, we recover the standard dis-

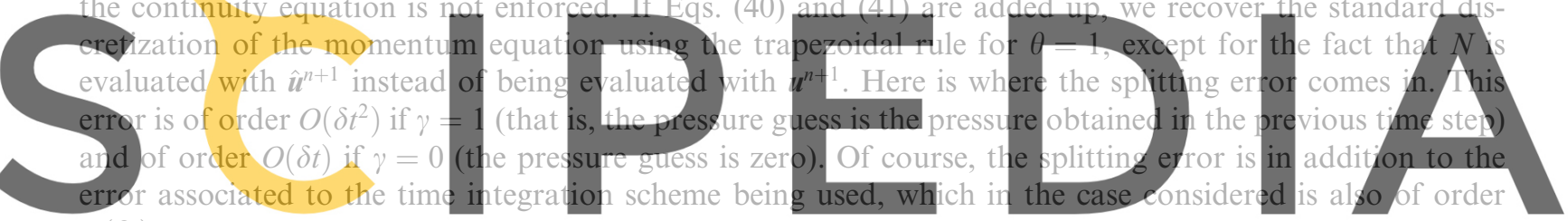

$O(\delta t)$.

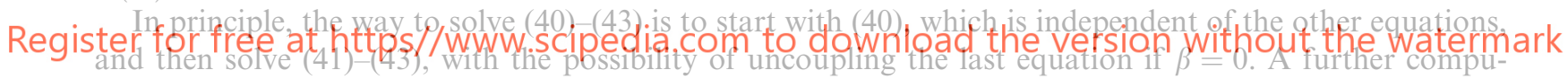

tational advantage can be achieved if we take the divergence of (41) and use the modified continuity

Eq. (42). This leads to

$$
\left(1+\frac{\alpha^{2}}{\delta t}\right) \Delta p^{n+1}=\frac{1}{\delta t} \nabla \cdot \hat{\boldsymbol{u}}^{n+1}+\gamma \Delta p^{n}+\frac{\alpha}{\delta t} \nabla \cdot \xi^{n+\beta}
$$

For $\beta=0$, this allows to solve, successively, (40) for $\hat{\boldsymbol{u}}^{n+1}$, (44) for $p^{n+1}$, (43) for $\xi^{n+1}$ and finally (41) for $\boldsymbol{u}^{n+1}$. This fact is extremely attractive from the computational standpoint and is probably the main reason for using fractional step or splitting techniques (see [15] for a review of such methods). However, there is a point that we have deliverately omitted, which is the treatment of the boundary conditions. This is irrelevant for our purpose of showing how to apply the stabilization method to this type of schemes.

Once the finite element approximation of the problem has been performed, the final algebraic system of equations to be solved is (33)-(36), which is obtained from the discretization of Eqs. (40), (44), (43) and (41). Now the parameters $\alpha_{K}$ can be considered again as defined element by element and take the resulting formulation as the proposed stabilized fractional step finite element method.

\subsubsection{Discrete variational form}

The matrix system given by (33)-(36) corresponds to the following discrete variational equations:

$$
\frac{1}{\delta t}\left(\hat{\boldsymbol{u}}_{h}^{n+1}-\boldsymbol{u}_{h}^{n}, \boldsymbol{v}_{h}\right)+c\left(\hat{\boldsymbol{u}}_{h}^{n+\theta}, \hat{\boldsymbol{u}}_{h}^{n+\theta}, \boldsymbol{v}_{h}\right)+a\left(\hat{\boldsymbol{u}}_{h}^{n+\theta}, \boldsymbol{v}_{h}\right)-\gamma b\left(p_{h}^{n}, \boldsymbol{v}_{h}\right)=\left(\boldsymbol{f}^{n+1}, \boldsymbol{v}_{h}\right),
$$




$$
\begin{aligned}
& \sum_{K}\left(\alpha_{K}^{2} \nabla p_{h}^{n+1}-\alpha_{K} \xi_{h}^{n+\beta}, \nabla q_{h}\right)_{K}+\sum_{K} \delta t\left(\nabla p_{h}^{n+1}-\gamma \nabla p_{h}^{n}, \nabla q_{h}\right)_{K}+b\left(q_{h}, \hat{\boldsymbol{u}}_{h}^{n+1}\right)=0, \\
& -\sum_{K} \alpha_{K}\left(\nabla p_{h}^{n+1}, \boldsymbol{\eta}_{h}\right)_{K}+\left(\boldsymbol{\xi}_{h}^{n+1}, \boldsymbol{\eta}_{h}\right)=0, \\
& \frac{1}{\delta t}\left(\boldsymbol{u}_{h}^{n+1}-\hat{\boldsymbol{u}}_{h}^{n+1}, \boldsymbol{v}_{h}\right)-b\left(p_{h}^{n+1}-\gamma p_{h}^{n}, \boldsymbol{v}_{h}\right)=0,
\end{aligned}
$$

which must be satisfied for all $\left(\boldsymbol{v}_{h}, q_{h}, \boldsymbol{\eta}_{h}\right) \in \boldsymbol{V}_{h, 0} \times Q_{h} \times \boldsymbol{V}_{h}$. This variational form of the problem allows us to draw some conclusions.

Let us consider first the case $\alpha_{K}=0$, that is, the classical fractional step scheme. In the case $\gamma=0$, it is seen from (46) that a pressure Laplacian multiplied by $\delta t$ is introduced in the incompressibility equation. This is the reason why the resulting scheme allows to use equal velocity-pressure interpolation, an observation found for example in [16-19]. In [20], the scheme is shown to be stable by considering it as a (perturbed) problem for the intermediate velocity $\hat{\boldsymbol{u}}_{h}^{n+1}$ instead of a problem for the end-of-step velocity $\boldsymbol{u}_{h}^{n+1}$. However, the fact that the stability (and therefore the convergence) of the scheme relies on the time step size $\delta t$ has severe drawbacks. If $\delta t$ is too large, the incompressiblity constraint will be poorly approximated, whereas if is too small there will be a lack of stability. From the analysis presented for the stationary case it follows that $\delta t$ must be of order $\mathrm{O}\left(h^{2}\right)$ to have both good stability and accuracy. It is known that good results are obtained if $\delta t$ is computed as the critical time step of the explicit approximation to the equations (that is, $\theta=0$ ).

The case $\gamma=1$ leads to a scheme in which the splitting error is of second order, and therefore with a better approximation to the incompressibility of the flow. However, in this case it is observed from (46) that stability may deteriorate as the steady state is reached, that is, as $p_{h}^{n+1}-p_{h}^{n} \rightarrow 0$ (in a certain norm). In order

\section{to prove convergence of the}

velocity-pressure spaces must

The use of $\alpha_{K}$ satisfying dondition

rely any more on the time step

and keeping the most

equations and the possibility of using equal velocity-pressure interpolations.

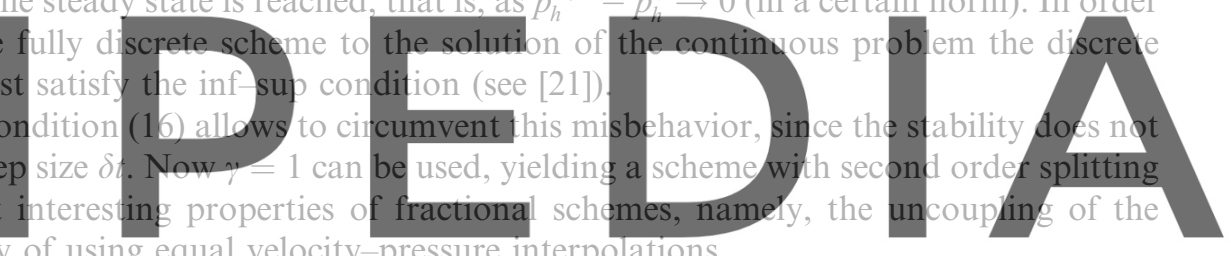

Register for free at https//www.scipedia.com to download the version without the watermark

\section{A stability estimate}

The purpose of this section is to prove a stability estimate for a simplified case of the fully discrete problem (21)-(23). The simplifications that will be assumed are that we shall take $\alpha_{K}$ equal for all the elements to a parameter $\alpha$ and that we shall take $\theta=1$. These assumptions are not restrictive if, as in our case, the purpose of the analysis is to get insight on the stability nature of the scheme analyzed.

The problem that we consider is therefore

$$
\begin{aligned}
& \left(\frac{\delta \boldsymbol{u}_{h}^{n}}{\delta t}, \boldsymbol{v}_{h}\right)+c\left(\boldsymbol{u}_{h}^{n+1}, \boldsymbol{u}_{h}^{n+1}, \boldsymbol{v}_{h}\right)+a\left(\boldsymbol{u}_{h}^{n+1}, \boldsymbol{v}_{h}\right)-b\left(p_{h}^{n+1}, \boldsymbol{v}_{h}\right)=\left(\boldsymbol{f}^{n+1}, \boldsymbol{v}_{h}\right), \\
& \alpha^{2}\left(\nabla p_{h}^{n+1}, \nabla q_{h}\right)-\alpha\left(\xi_{h}^{n+\beta}, \nabla q_{h}\right)+b\left(q_{h}, \boldsymbol{u}_{h}^{n+1}\right)=0, \\
& -\alpha\left(\nabla p_{h}^{n+1}, \boldsymbol{\eta}_{h}\right)+\left(\xi_{h}^{n+1}, \boldsymbol{\eta}_{h}\right)=0,
\end{aligned}
$$

for all $\left(\boldsymbol{v}_{h}, q_{h}, \boldsymbol{\eta}_{h}\right) \in \boldsymbol{V}_{h, 0} \times Q_{h} \times \boldsymbol{V}_{h}$. As it is seen from (50), we still consider the possibility of treating the pressure gradient projection either implicitly or explicitly.

We shall also make an assumption on the finite element mesh, namely, that is is quasi-uniform and of diameter $h$. In this case, the inverse estimates 


$$
\left\|\nabla \boldsymbol{v}_{h}\right\| \leqslant \frac{C_{\mathrm{inv}}}{h}\left\|\boldsymbol{v}_{h}\right\|, \quad\left\|\boldsymbol{v}_{h}\right\|_{L^{\infty}(\Omega)} \leqslant \frac{C_{\mathrm{inv}}}{h^{d / 2}}\left\|\boldsymbol{v}_{h}\right\| \quad \forall \boldsymbol{v}_{h} \in \boldsymbol{V}_{h}
$$

hold true. Again, the same results as for the steady-state case can be proved only assuming that the mesh is non-degenerate, but this does not introduce any further insight on the method.

From now on we use $C$, possibly with subscripts, to denote positive constants independent of $h$ and $\delta t$, not necessarily the same at different occurrences.

The idea is to obtain a stability estimate for the sequence of velocities $\boldsymbol{u}_{h}^{n}$ as well as for the sequence $\nabla^{h} p_{h}^{n}=h \nabla p_{h}^{n}$. In order to write down these estimates, let us introduce the following definition. If $\left\{f^{n}\right\}_{n=0}^{N}$ is a sequence of functions in a Banach space $X$ with norm $\|\cdot\|_{X}$, we say that

$$
\left\{f^{n}\right\} \in \ell^{p}(X) \quad \text { iff } \quad \sum_{n=0}^{N}\left\|f^{n}\right\|_{X}^{p} \delta t \leqslant C<\infty,
$$

for $1 \leqslant p<\infty$, and

$$
\left\{f^{n}\right\} \in \ell^{\infty}(X) \text { iff } \max _{n=0}\left\{\left\|f^{n}\right\|_{X}\right\} \leqslant C<\infty .
$$

\section{Our main stability result for problem (49)-(51) the following:}

Theorem 3. Suppose that the family of finite element partitions $\left\{\mathscr{T}_{h}\right\}_{h>0}$ is quasi-uniform and such that (15) holds. If $\alpha$ satisfies (16), then the solution to problem (49)-(51) verifies

$\left\{\boldsymbol{u}_{h}^{n}\right\} \in \ell^{\infty}\left(\boldsymbol{L}^{2}(\Omega)\right) \cap \ell^{2}\left(\boldsymbol{H}^{1}(\Omega)\right)$,

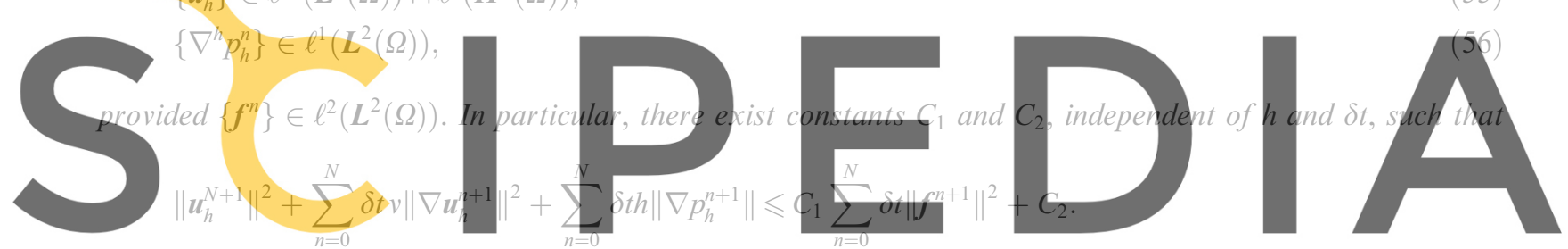

Register for free at https//www.scipedia.com, to download the version without the watermark

$$
\boldsymbol{g}_{i}^{n}:=P_{h, i}\left(\alpha \nabla p_{h}^{n}\right), \quad i=1,2,3 .
$$

From (51) we have that

$$
\xi_{h}^{n+1}=\boldsymbol{g}_{1}^{n+1}+\boldsymbol{g}_{2}^{n+1},
$$

and therefore the continuity equation can be written as

$$
\alpha\left(\boldsymbol{g}_{3}^{n+1}, \nabla q_{h}\right)+\bar{\beta} \alpha\left(\xi_{h}^{n+1}-\xi_{h}^{n}, \nabla q_{h}\right)+b\left(q_{h}, \boldsymbol{u}_{h}^{n+1}\right)=0,
$$

where $\bar{\beta}:=1-\beta$. We will be interested only in the cases $\bar{\beta}=0$ and $\bar{\beta}=1$. Adding up (57) with the momentum equation (49) taking $\boldsymbol{v}_{h}=\boldsymbol{u}_{h}^{n+1}$ and $q_{h}=p_{h}^{n+1}$ it is found that

$$
\left(\boldsymbol{u}_{h}^{n+1}, \frac{\delta \boldsymbol{u}_{h}^{n}}{\delta t}\right)+v\left\|\nabla \boldsymbol{u}_{h}^{n+1}\right\|^{2}+\left\|\boldsymbol{g}_{3}^{n+1}\right\|^{2}+\bar{\beta}\left(\xi_{h}^{n+1}-\xi_{h}^{n}, \alpha \nabla p_{h}^{n+1}\right)=\left(\boldsymbol{f}^{n+1}, \boldsymbol{u}_{h}^{n+1}\right)
$$

Taking into account that

$$
\begin{aligned}
\left(\xi_{h}^{n+1}-\xi_{h}^{n}, \alpha \nabla p_{h}^{n+1}\right) & =\left(\xi_{h}^{n+1}-\xi_{h}^{n}, \xi_{h}^{n+1}\right)=\frac{1}{2}\left\|\xi_{h}^{n+1}\right\|^{2}-\frac{1}{2}\left\|\xi_{h}^{n}\right\|^{2}+\frac{1}{2}\left\|\xi_{h}^{n+1}-\xi_{h}^{n}\right\|^{2}, \\
\left(\boldsymbol{f}^{n+1}, \boldsymbol{u}_{h}^{n+1}\right) & \leqslant C\left\|\boldsymbol{f}^{n+1}\right\|^{2}+\frac{v}{2}\left\|\nabla \boldsymbol{u}_{h}^{n+1}\right\|^{2}
\end{aligned}
$$


it follows from (58) that

$$
\begin{gathered}
\left\|\boldsymbol{u}_{h}^{n+1}\right\|^{2}-\left\|\boldsymbol{u}_{h}^{n}\right\|^{2}+\left\|\boldsymbol{u}_{h}^{n+1}-\boldsymbol{u}_{h}^{n}\right\|^{2}+\delta t v\left\|\nabla \boldsymbol{u}_{h}^{n+1}\right\|^{2}+\delta t\left\|\boldsymbol{g}_{3}^{n+1}\right\|^{2} \\
+\delta t \bar{\beta}\left(\left\|\xi_{h}^{n+1}\right\|^{2}-\left\|\xi_{h}^{n}\right\|^{2}+\left\|\xi_{h}^{n+1}-\xi_{h}^{n}\right\|^{2}\right) \leqslant C \delta t\left\|\boldsymbol{f}^{n+1}\right\|^{2}
\end{gathered}
$$

and, adding these equations up from $n=0$ to the last time interval $N$ it follows that

$$
\begin{aligned}
& \left\|\boldsymbol{u}_{h}^{N+1}\right\|^{2}+\sum_{n=0}^{N}\left\|\boldsymbol{u}_{h}^{n+1}-\boldsymbol{u}_{h}^{n}\right\|^{2}+\sum_{n=0}^{N} \delta t v\left\|\nabla \boldsymbol{u}_{h}^{n+1}\right\|^{2}+\sum_{n=0}^{N} \delta t\left\|\boldsymbol{g}_{3}^{n+1}\right\|^{2} \\
& \quad+\delta t \bar{\beta}\left(\left\|\xi_{h}^{N+1}\right\|^{2}+\sum_{n=0}^{N}\left\|\xi_{h}^{n+1}-\boldsymbol{\xi}_{h}^{n}\right\|^{2}\right) \leqslant C_{1} \sum_{n=0}^{N} \delta t\left\|\boldsymbol{f}^{n+1}\right\|^{2}+C_{2} .
\end{aligned}
$$

Of course this is also true for any $N_{0} \leqslant N$ and proves (55). To prove the stability estimate for the pressure, observe from (59) that $\boldsymbol{g}_{3}^{n}$ is already bounded in $\ell^{2}\left(\boldsymbol{L}^{2}(\Omega)\right)$, and therefore it is also bounded in $\ell^{1}\left(\boldsymbol{L}^{2}(\Omega)\right)$. Assuming that condition (15) holds, it only remains to obtain a bound for $\boldsymbol{g}_{1}^{n}$. For that, let us take $\boldsymbol{v}_{h}=P_{h, 1}\left(\nabla p_{h}^{n+1}\right)$ in the momentum equation. Using the inverse estimate (52) and Schwarz's inequality we obtain

$$
\begin{aligned}
\left\|P_{h, 1}\left(\nabla p_{h}^{n+1}\right)\right\|^{2}=- & \left(P_{h, 1}\left(\nabla p_{h}^{n+1}\right), \frac{\delta \boldsymbol{u}_{h}^{n}}{\delta t}\right)+\left(\boldsymbol{f}^{n+1}, P_{h, 1}\left(\nabla p_{h}^{n+1}\right)\right) \\
& -a\left(\boldsymbol{u}_{h}^{n+1}, P_{h, 1}\left(\nabla p_{h}^{n+1}\right)\right)-c\left(\boldsymbol{u}_{h}^{n+1}, \boldsymbol{u}_{h}^{n+1}, P_{h, 1}\left(\nabla p_{h}^{n+1}\right)\right) \\
\leqslant & \left\|P_{h, 1}\left(\nabla p_{h}^{n+1}\right)\right\|\left\|\frac{\delta \boldsymbol{u}_{h}^{n}}{\delta t}\right\|+\left\|\boldsymbol{f}^{n+1}\right\|\left\|P_{h, 1}\left(\nabla p_{h}^{n+1}\right)\right\| \\
& +\left(N_{a}\left\|\boldsymbol{u}_{h}^{n+1}\right\|+N_{c}\left\|\boldsymbol{u}_{h}^{n+1}\right\|^{2}\right) \frac{C_{\text {inv }}}{h}\left\|P_{h, 1}\left(\nabla p_{h}^{n+1}\right)\right\| .
\end{aligned}
$$

Dividing both sides by $P_{h, 1}\left(\nabla p_{h}^{n+1}\right)$ (assuming it is $\neq 0$ ), multiplying by $h \delta t$ and adding the resulting equations for $n=0,1, \ldots, N$ it follows that

$$
\sum_{n=0}^{N} \delta t h\left\|P_{h, 1}\left(\nabla p_{h}^{n+1}\right)\right\| \leqslant \sum_{n=0}^{N}\left\|\boldsymbol{u}_{h}^{n+1}-\boldsymbol{u}_{h}^{n}\right\|+\sum_{n=0}^{N} \delta t\left\|\boldsymbol{f}^{n+1}\right\|+\sum_{n=0}^{N} \delta t N_{a}\left\|\boldsymbol{u}_{h}^{n+1}\right\|+\sum_{n=0}^{N} \delta t N_{c}\left\|\boldsymbol{u}_{h}^{n+1}\right\|^{2} .
$$

The bound for $\boldsymbol{g}_{1}^{n}$ follows from the behavior assumed for $\alpha$ and from estimate (59). This proves (56).

The stability estimate for the pressure gradient projection given by (56) can be improved in some cases, although not in the general case of the 3D Navier-Stokes equations. This is what the following result states.

Theorem 4. Under the same conditions as in Theorem 3, suppose also that either $c=0$ (Stokes problem) or $d=2\left(2 D\right.$ flows). In the case $\beta=0$ suppose also that $\delta t \geqslant C h^{2}$. Then the solution to problem (49)-(51) verifies the stability estimate

$$
\left\{\nabla^{h} p_{h}^{n}\right\} \in \ell^{2}\left(\boldsymbol{L}^{2}(\Omega)\right)
$$

Proof. Let us consider now the continuity Eq. (57) for time step $n$ and $n+1$. Subtracting both it is found that

$$
\frac{\alpha}{\delta t}\left(\boldsymbol{g}_{3}^{n+1}-\boldsymbol{g}_{3}^{n}, \nabla q_{h}\right)+\bar{\beta} \frac{\alpha}{\delta t}\left(\xi_{h}^{n+1}-2 \xi_{h}^{n}+\xi_{h}^{n-1}, \nabla q_{h}\right)-\left(\nabla q_{h}, \frac{\delta \boldsymbol{u}_{h}^{n}}{\delta t}\right)=0
$$


Taking now $q_{h}=p_{h}^{n+1}$ we get

$$
\begin{aligned}
\left(P_{h, 1}\left(\nabla p_{h}^{n+1}\right), \frac{\delta \boldsymbol{u}_{h}^{n}}{\delta t}\right)= & \frac{1}{\delta t}\left(\boldsymbol{g}_{3}^{n+1}-\boldsymbol{g}_{3}^{n}, \boldsymbol{g}_{3}^{n+1}\right)+\bar{\beta} \frac{1}{\delta t}\left(\xi_{h}^{n+1}-2 \xi_{h}^{n}+\xi_{h}^{n-1}, \xi_{h}^{n+1}\right) \\
= & \frac{1}{2 \delta t}\left(\left\|\boldsymbol{g}_{3}^{n+1}\right\|^{2}-\left\|\boldsymbol{g}_{3}^{n}\right\|^{2}+\left\|\boldsymbol{g}_{3}^{n+1}-\boldsymbol{g}_{3}^{n}\right\|^{2}\right) \\
& +\bar{\beta} \frac{1}{2 \delta t}\left(\left\|\xi_{h}^{n+1}\right\|^{2}+\left\|\xi_{h}^{n-1}\right\|^{2}-\left\|2 \xi_{h}^{n}\right\|^{2}-\left\|2 \xi_{h}^{n}-\xi_{h}^{n-1}\right\|^{2}\right. \\
& \left.+\left\|\xi_{h}^{n+1}-2 \xi_{h}^{n}+\xi_{h}^{n-1}\right\|^{2}\right) .
\end{aligned}
$$

Taking now $\boldsymbol{v}_{h}=P_{h, 1}\left(\nabla p_{h}^{n+1}\right)$ in the momentum equation, using the continuity of $a$ in $\boldsymbol{H}^{1}(\Omega)^{2}$ and the continuity of $c$ in $\boldsymbol{L}^{2}(\Omega) \times \boldsymbol{H}^{1}(\Omega) \times \boldsymbol{L}^{\infty}(\Omega)$ and the inverse estimates in (52) it follows that

$$
\begin{aligned}
\left(P_{h, 1}\left(\nabla p_{h}^{n+1}\right), \frac{\delta \boldsymbol{u}_{h}^{n}}{\delta t}\right)+ & \left\|P_{h, 1}\left(\nabla p_{h}^{n+1}\right)\right\|^{2} \\
= & \left(\boldsymbol{f}^{n+1}, P_{h, 1}\left(\nabla p_{h}^{n+1}\right)\right) \\
& -a\left(\boldsymbol{u}_{h}^{n+1}, P_{h, 1}\left(\nabla p_{h}^{n+1}\right)\right)-c\left(\boldsymbol{u}_{h}^{n+1}, \boldsymbol{u}_{h}^{n+1}, P_{h, 1}\left(\nabla p_{h}^{n+1}\right)\right) \\
\leqslant & \left\|\boldsymbol{f}^{n+1}\right\|\left\|P_{h, 1}\left(\nabla p_{h}^{n+1}\right)\right\|+N_{a}\left\|\boldsymbol{u}_{h}^{n+1}\right\|\left\|P_{h, 1}\left(\nabla p_{h}^{n+1}\right)\right\| \\
& +N_{c}\left\|\boldsymbol{u}_{h}^{n+1}\right\|\left\|\boldsymbol{u}_{h}^{n+1}\right\|\left\|P_{h, 1}\left(\nabla p_{h}^{n+1}\right)\right\|_{L^{\infty}(\Omega)} \\
\leqslant & \left\|\boldsymbol{f}^{n+1}\right\|\left\|P_{h, 1}\left(\nabla p_{h}^{n+1}\right)\right\| \\
& +C\left\|\boldsymbol{u}_{h}^{n+1}\right\|\left(\frac{N_{a}}{h}+\frac{N_{c}}{h^{d / 2}}\left\|\boldsymbol{u}_{h}^{n+1}\right\|\right)\left\|P_{h, 1}\left(\nabla p_{h}^{n+1}\right)\right\| .
\end{aligned}
$$

Since the velocities are in $\ell^{\infty}\left(\boldsymbol{L}^{2}(\Omega)\right)$, the norm inside the parenthesis is bounded. Also, either if $N_{c}=0$ (Stokes problem) or if $d=2$ this parenthesis behaves as $1 / h$. Therefore, from Young's and Poincare's inequalities we have

$$
\left(P_{h, 1}\left(\nabla p_{h}^{n+1}\right), \frac{\delta \boldsymbol{u}_{h}^{n}}{\delta t}\right)+\left\|P_{h, 1}\left(\nabla p_{h}^{n+1}\right)\right\|^{2} \leqslant C\left\|\boldsymbol{f}^{n+1}\right\|^{2}+C \frac{v}{h^{2}}\left\|\nabla \boldsymbol{u}_{h}^{n+1}\right\|^{2}+\frac{1}{2}\left\|P_{h, 1}\left(\nabla p_{h}^{n+1}\right)\right\|^{2},
$$

and, using (62) and multiplying by $h^{2} \delta t$,

$$
\begin{aligned}
& \delta t h^{2}\left\|P_{h, 1}\left(\nabla p_{h}^{n+1}\right)\right\|^{2}+h^{2}\left(\left\|\boldsymbol{g}_{3}^{n+1}\right\|^{2}-\left\|\boldsymbol{g}_{3}^{n}\right\|^{2}+\left\|\boldsymbol{g}_{3}^{n+1}-\boldsymbol{g}_{3}^{n}\right\|^{2}\right) \\
& +\bar{\beta} h^{2}\left(\left\|\boldsymbol{\xi}_{h}^{n+1}\right\|^{2}+\left\|\xi_{h}^{n-1}\right\|^{2}-\left\|2 \boldsymbol{\xi}_{h}^{n}\right\|^{2}-\left\|2 \xi_{h}^{n}-\xi_{h}^{n-1}\right\|^{2}+\left\|\xi_{h}^{n+1}-2 \xi_{h}^{n}+\xi_{h}^{n-1}\right\|^{2}\right) \\
& \leqslant C_{1} \delta t\left\|\boldsymbol{f}^{n+1}\right\|^{2}+C_{2} \delta t\left\|v \nabla \boldsymbol{u}_{h}^{n+1}\right\|^{2} .
\end{aligned}
$$

Adding up from $n=0$ up to $N$ (and neglecting some positive terms) we get

$$
\begin{aligned}
& \sum_{n=0}^{N} \delta t h^{2}\left\|P_{h, 1}\left(\nabla p_{h}^{n+1}\right)\right\|^{2}+\bar{\beta} h^{2}\left\|\boldsymbol{\xi}_{h}^{N+1}\right\|^{2} \\
& \quad \leqslant C_{1} \sum_{n=0}^{N} \delta t\left\|\boldsymbol{f}^{n+1}\right\|^{2}+C_{2} \sum_{n=0}^{N} \delta t\left\|v \nabla \boldsymbol{u}_{h}^{n+1}\right\|^{2}+\bar{\beta} h^{2}\left(\left\|\xi_{h}^{N}\right\|^{2}+2 \sum_{n=0}^{N}\left\|\xi_{h}^{n}-\xi_{h}^{n-1}\right\|^{2}\right)
\end{aligned}
$$

Finally, we can use condition (16) on $\alpha$ in (59) to obtain an estimate for the norm of $h P_{13}\left(\nabla p_{h}^{n+1}\right)$, which allows to obtain an estimate for the norm of the whole pressure gradient if the weak inf-sup condition (15) holds. The combination of (59) and (64) yields therefore

$$
\sum_{n=0}^{N} \delta t h^{2}\left\|\nabla p_{h}^{n+1}\right\|^{2} \leqslant\left(C_{1}+C_{2} \bar{\beta} \frac{h^{2}}{\delta t}\right)\left(\sum_{n=0}^{N} \delta t\left\|\boldsymbol{f}^{n+1}\right\|^{2}+C_{3}\right) .
$$

This completes the proof of the theorem. 
It is observed from Theorems 3 and 4 that each of the members of the sequence of velocities are $L^{2}$-stable, whereas the $L^{2}$ spatial norm of the velocity gradient and the pressure gradient (multiplied by $h$ ) are stable in the sense that the discrete approximation to their $L^{2}$ norm (or $L^{1}$ norm) in time is bounded. This is frequently expressed by saying that the velocities are strongly stable in $L^{2}$ and weakly in $H^{1}$, whereas the pressure gradient (multiplied by $h$ ) is weakly stable in $L^{2}$.

A further remark refers to the need for the condition $\delta t \geqslant C h^{2}$ in Theorem 4 when the projection of the pressure gradient is treated explicitly. This expresses the fact that the term added to the incompressility constraint must be small compared to $\delta t$. However, it is observed from (59) that without this assumption we already have control over the norm of $\boldsymbol{g}_{3}^{n+1}$ and only the norm of $P_{h, 1}\left(\nabla p_{h}^{n+1}\right)$ needs to be controlled (assuming that the weak inf-sup condition holds true). On the other hand, very few components of $\nabla p_{h}$ belong only to $\boldsymbol{V}_{h, 0}$ (none for linear and multilinear elements!) and thus a bound for $P_{h, 3}\left(\alpha \nabla p_{h}^{n+1}\right)$ implies stability for most of the pressure components. In practice, we have never observed the need for having $\delta t$ large enough and we have always obtained perfectly stable solutions when the projection of the pressure gradient is treated explicitly. Moreover, in Theorem 3 we have seen that no conditions on $\delta t$ are needed to obtain stability in $\ell^{1}\left(\boldsymbol{L}^{2}(\Omega)\right)$ for the pressure gradient.

\section{Numerical tests}

In this section we show the numerical results obtained with the stabilized, monolithic scheme (21)-(23) in two different problems, a test case with an analytical solution and the standard problem of the flow past a circular cylinder.

\subsection{A case with an analytical solution}

We consider the following analytical solution of the unsteady, incompressible Navier-Stokes equations in two dimensions, called the Taylor vortex problem (see [22,23]):

$$
\begin{aligned}
& u(x, y, t)=-\cos (\pi x) \sin (\pi y) \mathrm{e}^{-2 v \pi^{2} t} \\
& v(x, y, t)=\sin (\pi x) \cos (\pi y) \mathrm{e}^{-2 v \pi^{2} t} \\
& p(x, y, t)=(-1 / 4)(\cos (2 \pi x)+\cos (2 \pi y)) \mathrm{e}^{-4 v \pi^{2} t}
\end{aligned}
$$

where $v>0$ is the fluid's kinematic viscosity (which we took equal to 0.1 ). We employ this example to check the accuracy properties of the numerical scheme (21)-(23) with respect to the time discretization for different values of the parameter $\theta$. The case $\theta=1$ is studied analytically in [24], where we prove first order error estimates in the time step size and optimal order estimates in the mesh size with the given finite element interpolation for the fully discrete solution.

We took a computational domain consisting of the unit square $\Omega=[0,1] \times[0,1]$, and imposed timedependent, Dirichlet-type boundary conditions given by the value of the analytical solution (65) on the boundary of $\Omega$. We used a uniform $31 \times 31$ noded mesh to discretize the domain, and the same mesh points were used to define both a $Q_{1}$ and a $Q_{2}$ quadrilateral element interpolation, for comparison purposes.

We integrated the Navier-Stokes Eqs. (1)-(4) with the scheme (21)-(23) for $\theta=1$ and $\theta=1 / 2$ with different time step sizes, starting from (65) at $t=0$ as a velocity initial condition, to a final time $T=1$. We then calculated the $L^{2}$-norm of the velocity, the velocity gradient and the pressure gradient errors with respect to the analytical solution (65) at $t=1$, these are the variables the stability of which is established in Theorem 3 and for which error estimates are derived in [24]. For each value of $\theta$, we considered the cases $\beta=0,1$, corresponding to an explicit/implicit approximation of the pressure gradient. Also, when $\theta=1$ we considered the possibility of treating explicitly the advection velocity of the non-linear convective term within each time step, that is to say, to take this convective term as $c\left(\boldsymbol{u}_{h}^{n}, \boldsymbol{u}_{h}^{n+1}, \boldsymbol{v}_{h}\right)$. This also leads to a first order time integration scheme.

The backward Euler case $\theta=1$ displayed first order accuracy in all three variables and all four methods. The results obtained in this case with a $Q_{1}$ element interpolation can be seen in Figs. 1-3, where we plot the 


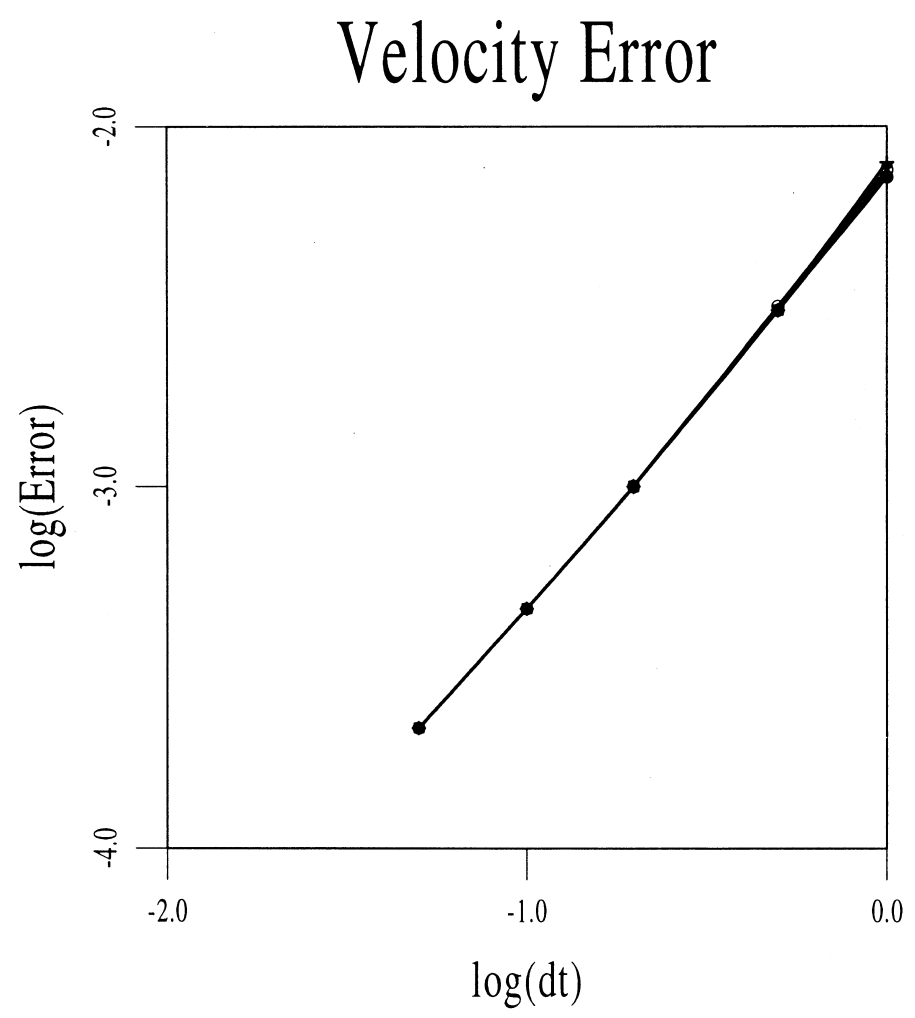

Fig. 1. Taylor flow, $\theta=1, Q_{1}$ element, velocity errors: $\circ \beta=0$, linearized scheme; $\beta=1$, linearized scheme; $+\beta=0$, non-linear scheme; $\times \beta=1$, non-linear scheme.

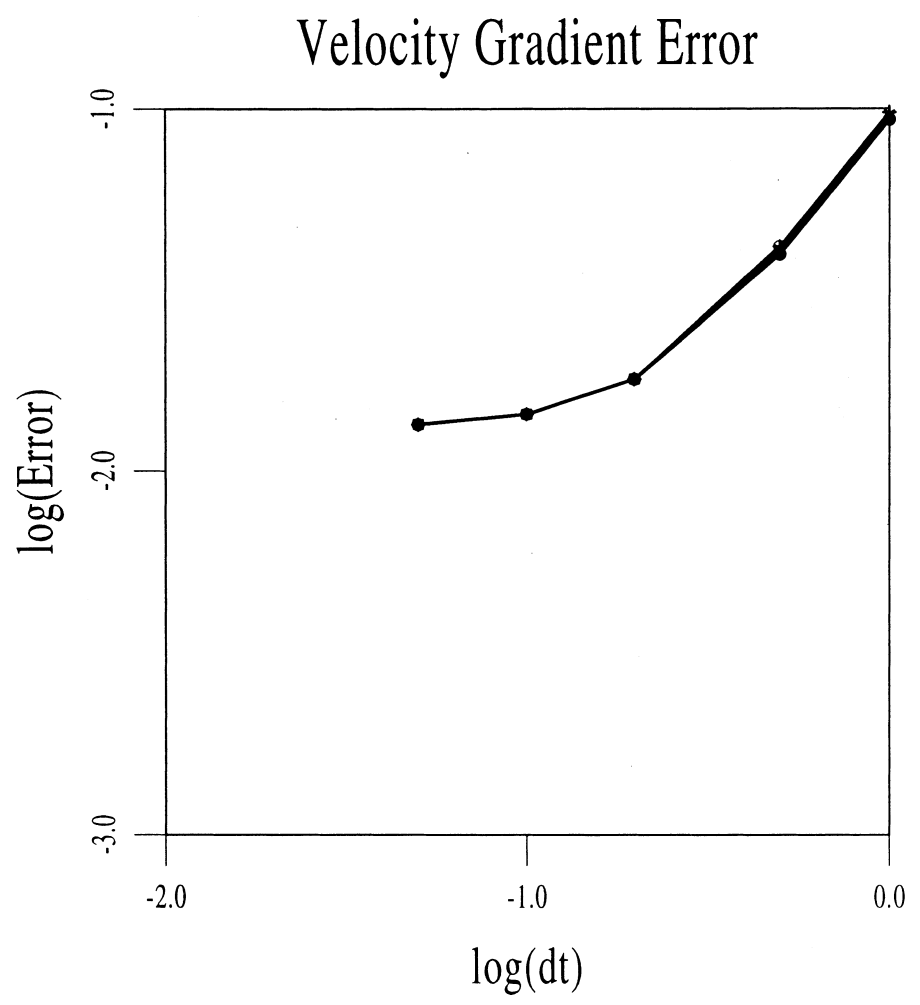

Fig. 2. Taylor flow, $\theta=1, Q_{1}$ element, velocity gradient errors: $\circ \beta=0$, linearized scheme; $\bullet \beta=1$, linearized scheme; $+\beta=0$, nonlinear scheme; $\times \beta=1$, non-linear scheme. 


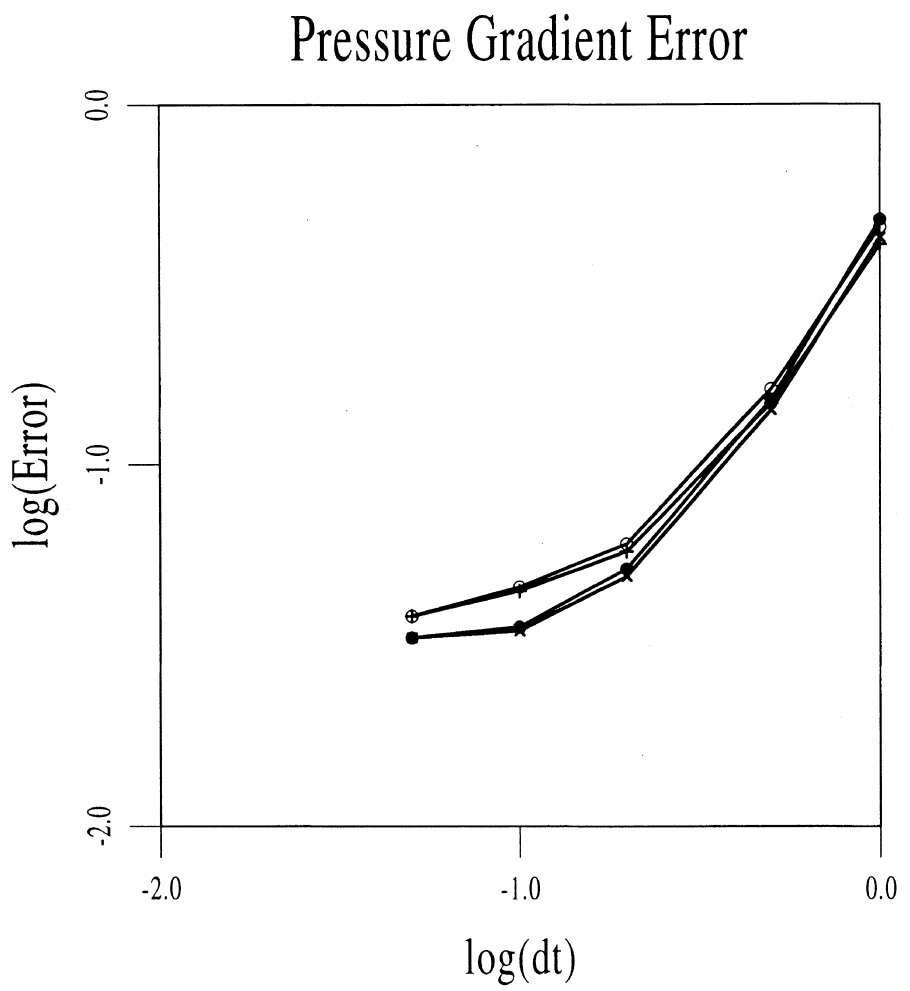

Fig. 3. Taylor flow, $\theta=1, Q_{1}$ element, pressure gradient errors: $\circ \beta=0$, linearized scheme; $\bullet \beta=1$, linearized scheme; $+\beta=0$, nonlinear scheme; $\times \beta=1$, non-linear scheme.

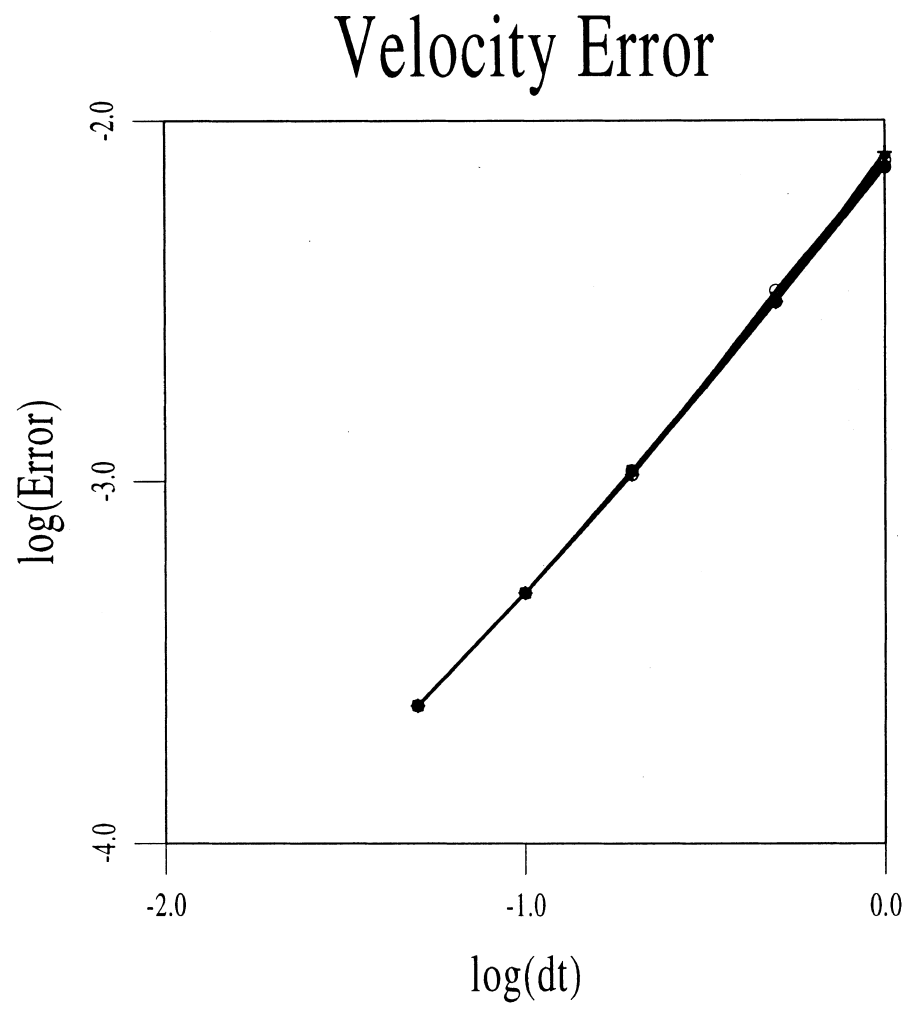

Fig. 4. Taylor flow, $\theta=1, Q_{2}$ element, velocity errors: $\circ \beta=0$, linearized scheme; $\bullet \beta=1$, linearized scheme; $+\beta=0$, non-linear scheme; $\times \beta=1$, non-linear scheme. 


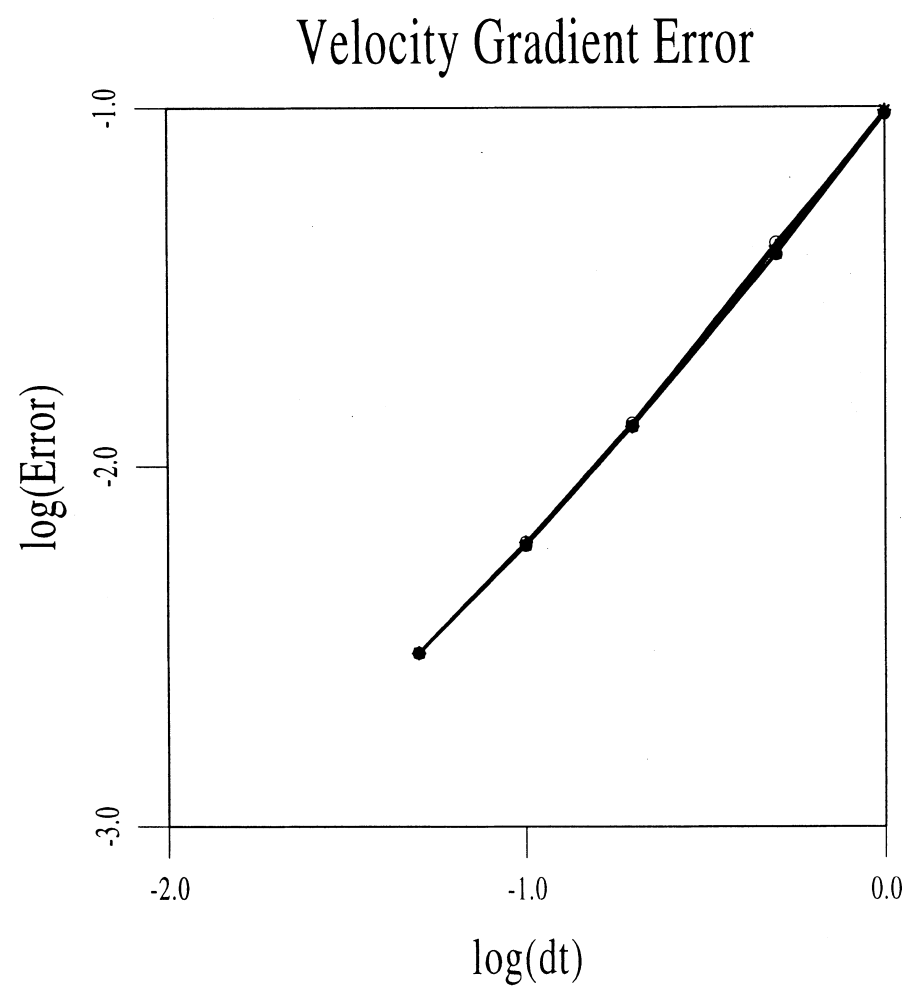

Fig. 5. Taylor flow, $\theta=1, Q_{2}$ element, velocity gradient errors: $\circ \beta=0$, linearized scheme; $\bullet \beta=1$, linearized scheme; $+\beta=0$, nonlinear scheme; $\times \beta=1$, non-linear scheme.

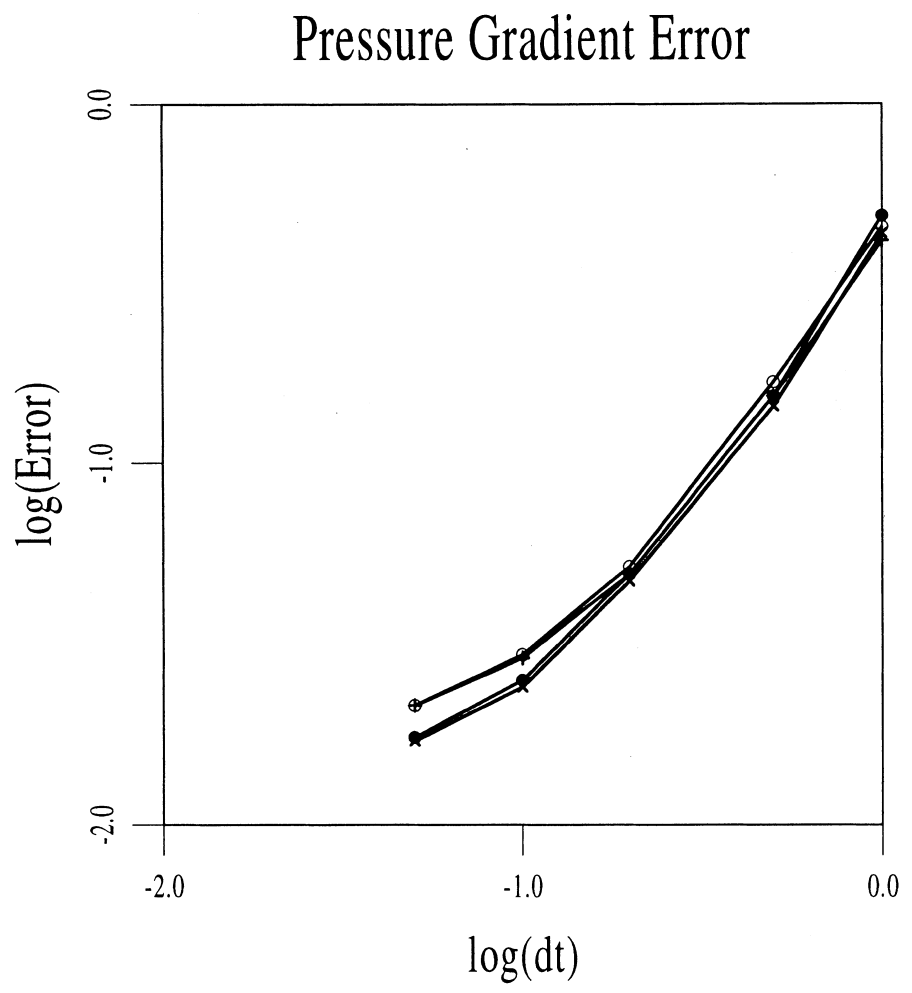

Fig. 6. Taylor flow, $\theta=1, Q_{1}$ element, pressure gradient errors: $\circ \beta=0$, linearized scheme; $\bullet \beta=1$, linearized scheme; $+\beta=0$, nonlinear scheme; $\times \beta=1$, non-linear scheme. 
different errors versus the time step size in logarithmic scale. However, first order accuracy is lost in the low $\delta t$ range for the velocity gradient and the pressure gradient, since the space error is reached then, the velocity gradient is shown in [24] to have $\mathrm{O}(h)$ spatial accuracy for the $Q_{1}$ element case while the pressure gradient is only bounded. For the mesh considered, we have $h \simeq 1 / 30$. The velocity spatial error, which is $\mathrm{O}\left(h^{2}\right)$, is not reached.

We then repeated the calculation with a $Q_{2}$ element interpolation; we plot the results in Figs. 4-6. First order time accuracy is also attained in this case. Moreover, the velocity gradient and pressure gradient errors confirmed their respective $\mathrm{O}\left(h^{2}\right)$ and $\mathrm{O}(h)$ theoretical estimates (see [24]).

The second order Crank-Nicholson case $\theta=1 / 2$ proved very sensitive to the values of the stabilization parameters $\alpha_{K}$. We finally took them as:

$$
\alpha_{K}=\left(\frac{4 v}{\alpha_{0} h_{K}^{2}}+\frac{2 v_{K}}{h_{K}}\right)^{-1 / 2},
$$

where $h_{K}$ is the size of element $K, v_{K}$ is a representative velocity of that element at the current time step and $\alpha_{0}$ is a given constant (which we took equal to $1 / 3$ for bilinear elements). These are the optimal values of the stabilization parameters of the GLS method. For large values of the viscosity and small element sizes, (66) behaves as condition (16) dictates. In general cases, we have found good results using (66).

Although not as sharp as in the previous case, it displayed second order accuracy for the velocity solution both in the $L^{2}$-norm and in the $H^{1}$-norm, and both for the $Q_{1}$ and $Q_{2}$ finite element spatial interpolations (in the first case, the spatial error also degraded the accuracy for low $\delta t$ values). The errors found in this case can be seen in Figs. 7 and 8, for the $Q_{1}$ element case, and Figs. 9 and 10 for the $Q_{2}$ element.

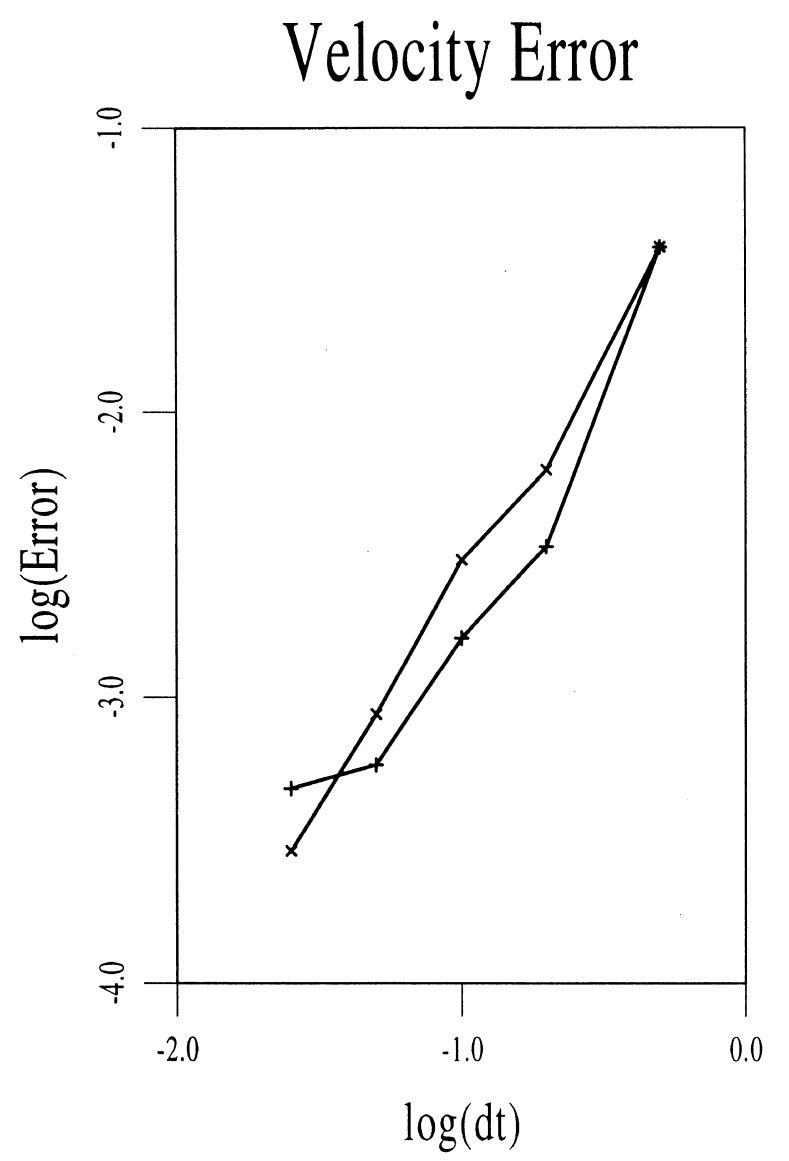

Fig. 7. Taylor flow, $\theta=1 / 2, Q_{1}$ element, velocity errors: $+\beta=0 ; \times \beta=1$. 


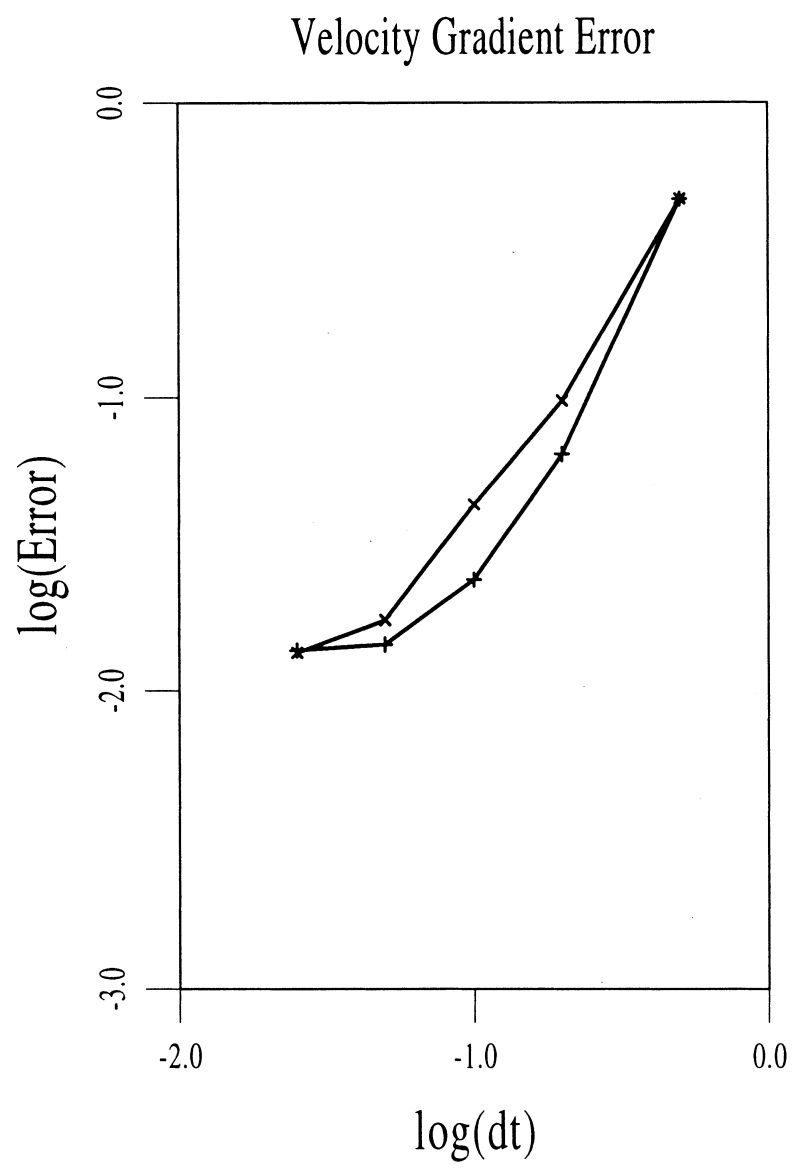

Fig. 8. Taylor flow, $\theta=1 / 2, Q_{1}$ element, velocity gradient errors: $+\beta=0 ; \times \beta=1$.

Finally, we plot the streamlines and pressure contours obtained at the end of one of the runs in Figs. 11 and 12 , respectively.

\subsection{Flow past a circular cylinder}

We then considered the problem of the flow past a circular cylinder, which has become a compulsory benchmark test for transient algorithms for the Navier-Stokes equations.

It is well known that for low values of the Reynolds number, the solution is steady and symmetric about a line parallel to the free-stream flow through a cylinder diameter; a pair of symmetrical eddies develop downstream of the cylinder. But beyond a critical value of $R e$ (which is larger than 40), the steady solution becomes unstable and a periodic solution develops, so that vortex shedding sets in.

The shedding period of the solution is one of the most studied quantities to compare quantitatively the results obtained in this problem. Equivalently, one can look at the Strouhal number or adimensional frequency, defined as $\mathrm{St}=D / u_{0} \tau, D$ being the cylinder diameter, $u_{0}$ the inflow velocity and $\tau$ the shedding period of the solution. It is now known that the shedding characteristics (and the Strouhal number, in particular) are significantly affected by the dissipation introduced by the time-stepping scheme, as well as by the spatial interpolation used (see [25], for instance). Moreover, the computed unsteady flow is also affected by the introduction of finite boundaries in the domain. While the location of the upstream (or inflow) and the downstream (or outflow) boundaries does not seem to affect the solution much (as long as the latter is further than 6 cylinder diameters, see [26]), the shedding solution is far more sensitive to the location of the lateral boundaries, as was recently found in [27]. In particular, the shedding period can become too small 


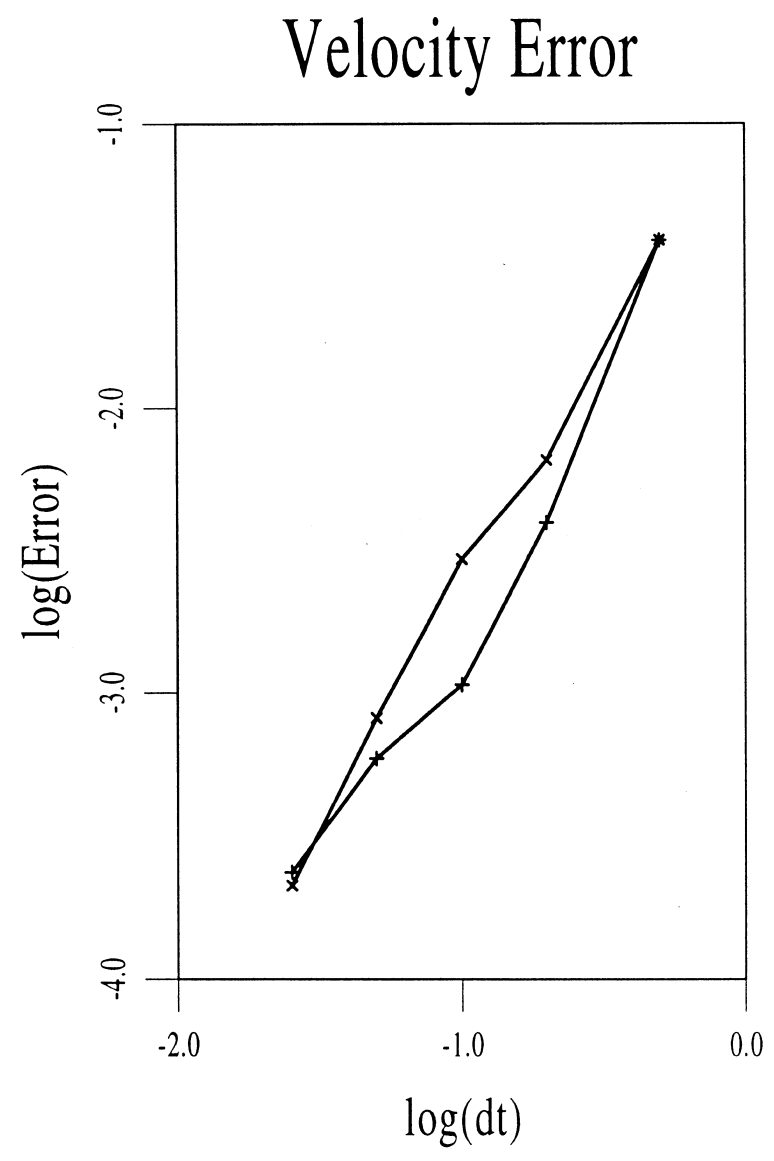

Fig. 9. Taylor flow, $\theta=1 / 2, Q_{2}$ element, velocity errors: $+\beta=0 ; \times \beta=1$.

when the lateral boundaries are placed too close to the cylinder. We placed these boundaries 12 cylinder diameters away form the cylinder, which according to [27] should be enough not to affect the solution.

Thus, we considered a cylinder of unit diameter and took a computational domain consisting of the rectangle $[0,50] \times[0,24]$, the center of the cylinder being situated at the point $(12 ., 12$.). The boundary conditions taken are the ones usually imposed in this problem, a unit free-stream horizontal velocity at the inflow, a solid wall condition on the cylinder surface, zero normal velocity and zero tangential stress on the lateral boundaries and a traction-free condition at the outflow. The mesh used in this case can be seen in Fig. 13; it consists of 3000 nodes and 2880 of the $Q_{1}$ elements.

We used the explicit pressure gradient, non-linear convection scheme (that is, (21)-(23) with $\beta=0$ ), with local values of the coefficients $\alpha$ given again by (66). At each time step, the non-linearity of the problem was solved by a Picard iteration with an error tolerance of $10^{-3}$ (for which 2 or 3 iterations were enough); the velocity-pressure systems of linear equations were solved by the iterative GMRES method, with an error tolerance of $10^{-5}$, while the pressure gradient system was solved by the conjugate gradient algorithm with a consistent mass matrix and the same error tolerance.

We first solved the problem for a Reynolds number of 40, which is based upon the free-stream velocity and the cylinder diameter, starting from the fluid at rest but for the prescribed boundary conditions. We performed 10 time steps of size $\delta t=0.5$ of the fully implicit scheme $\theta=1$ (so as to reach the steady state fastest), which yielded the steady solution very accurately.

We then raised the value of the Reynolds number to 100, we started the computation from the steady solution obtained for $R e=40$ and performed 10,000 steps of size $\delta t=0.1$ of the Crank-Nicholson method $\theta=1 / 2$. The solution started oscillating freely from the start, the final periodicity was reached by $t=750$. In this case, no artificial trick was needed to start up the periodic solution. In Fig. 14 we show the history of 


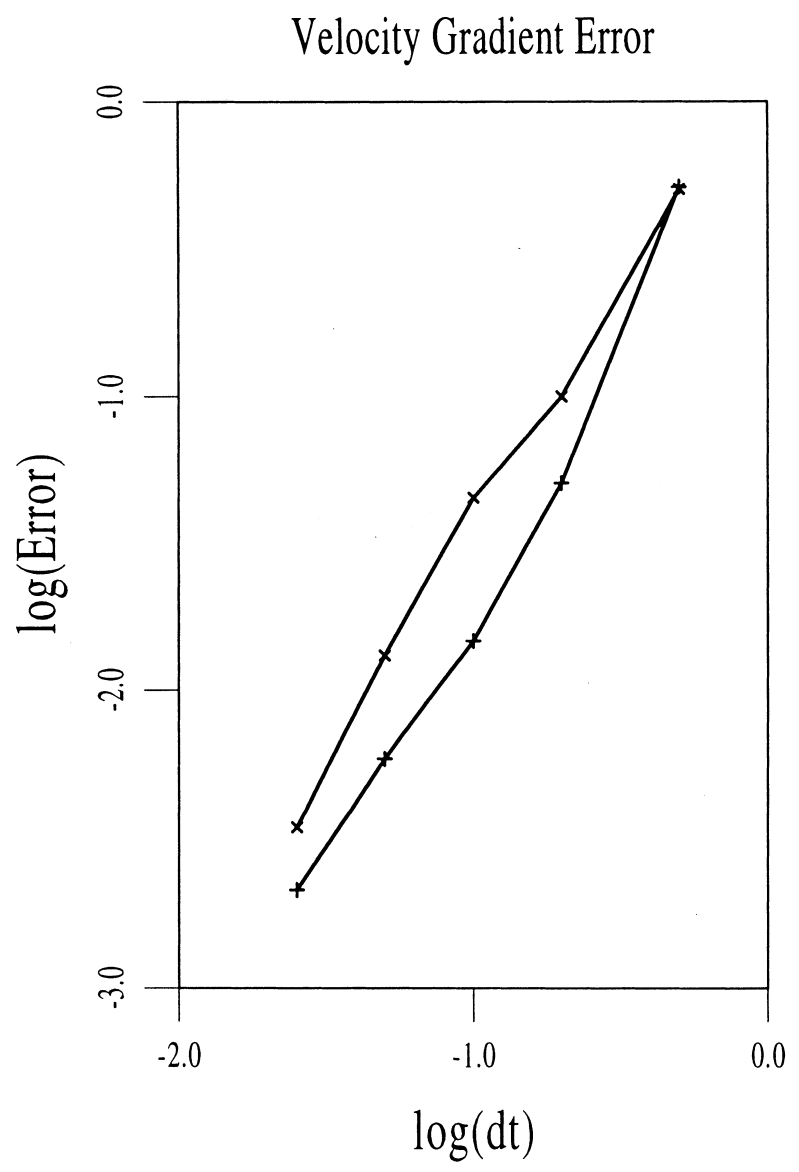

Fig. 10. Taylor flow, $\theta=1 / 2, Q_{2}$ element, velocity gradient errors: $+\beta=0 ; \times \beta=1$.

the horizontal velocity at a node situated at the point $(24.0,14$. $)$, that is, downstream of the cylinder and slightly higher. In order to improve the accuracy of the solution, we then continued the calculation with a time step size $\delta t=0.05$, with which we performed 500 steps. We finally found a Strouhal number of $S t=0.161$, or equivalently, a period of 6.2. To this order of precision, this is the same period that was found in [27] with a stabilized, space-time velocity-pressure formulation using a 7641 noded mesh (that is, more than two and a half times the number of nodes we used) in the present domain, a formulation which requieres of many more stabilization terms than ours. (A period of 6.0 was found in this reference with a stabilized velocity-pressure-stress formulation also using a Crank-Nicholson time integration scheme, with the same 7641 noded mesh and 3 extra coupled unknowns in 2 dimensions). It has to be said that most researchers who have solved this problem numerically up to now have considered a smaller domain (that is, one with lateral boundaries closer to the cylinder surface), this is probably the reason why smaller periods (between 5.8 and 6.0) have usually been found.

The streamlines obtained at the end of the computation are shown in Fig. 15. In Fig. 16 we plot the stationary streamlines; the wakes behind the cylinder can be clearly seen there. Finally, we show the pressure contours in Fig. 17. All these results compare very well with other published solutions (see [28,29], for instance).

\section{Conclusions}

In this paper we have presented the extension of a stabilization technique originally developed for steady problems to the transient case. The goal is to be able to use equal velocity-pressure interpolations, 


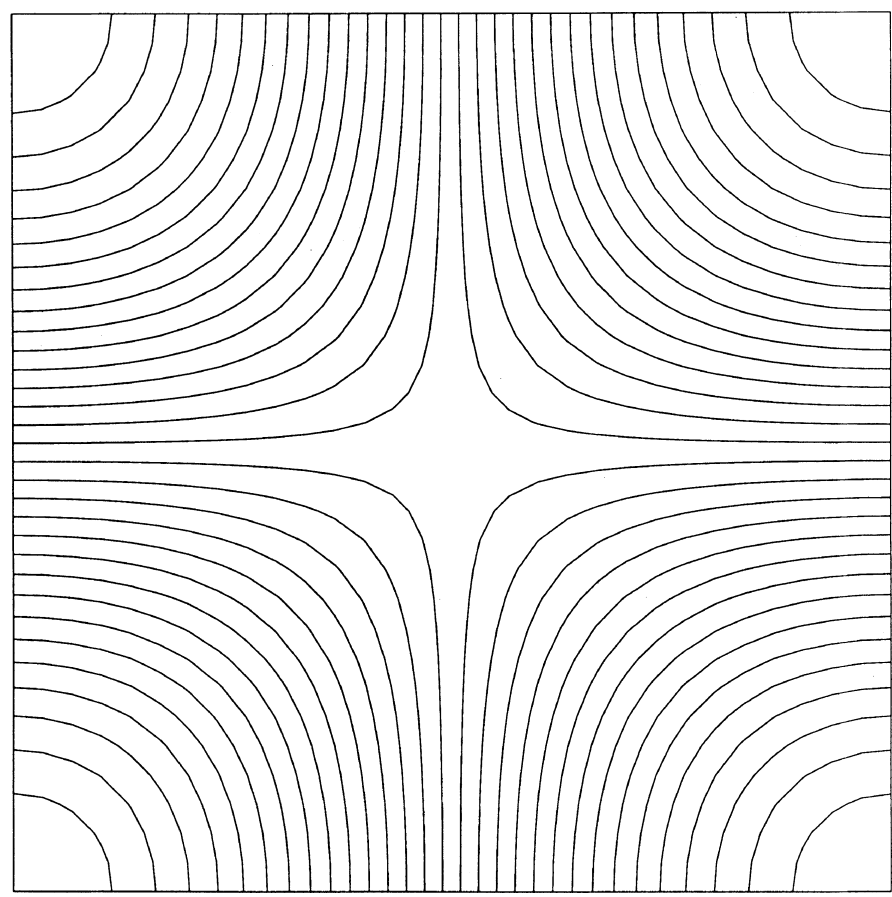

Fig. 11. Taylor flow, streamlines.

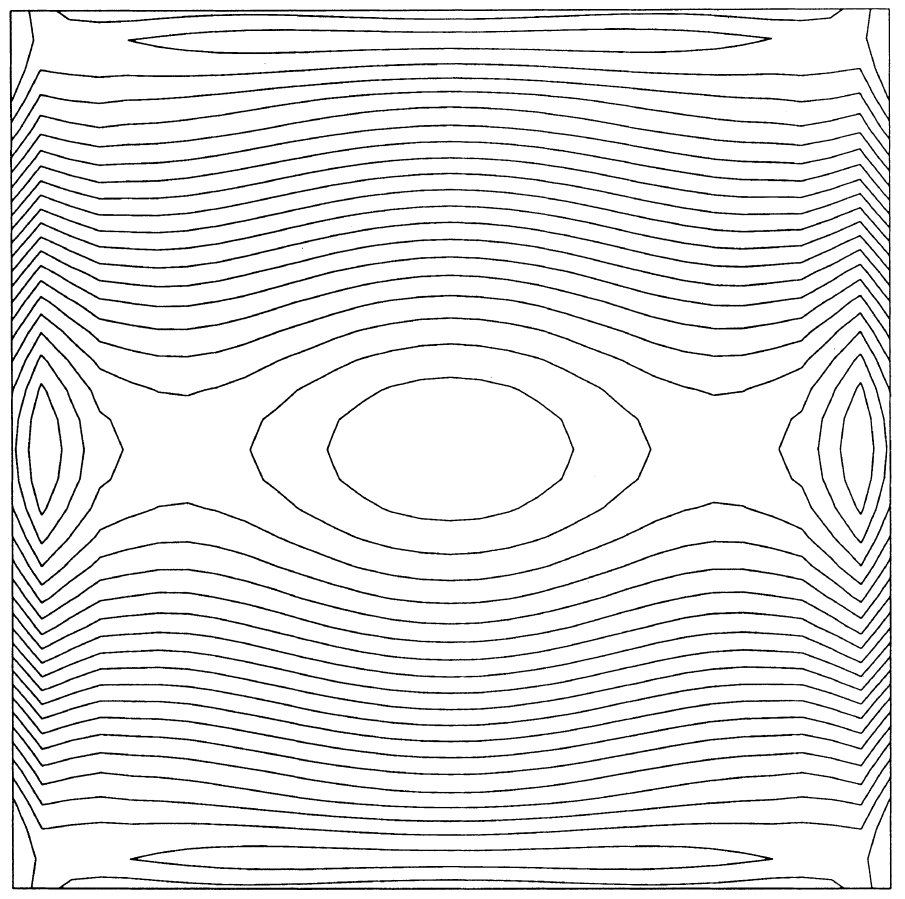

Fig. 12. Taylor flow, pressure contours. 


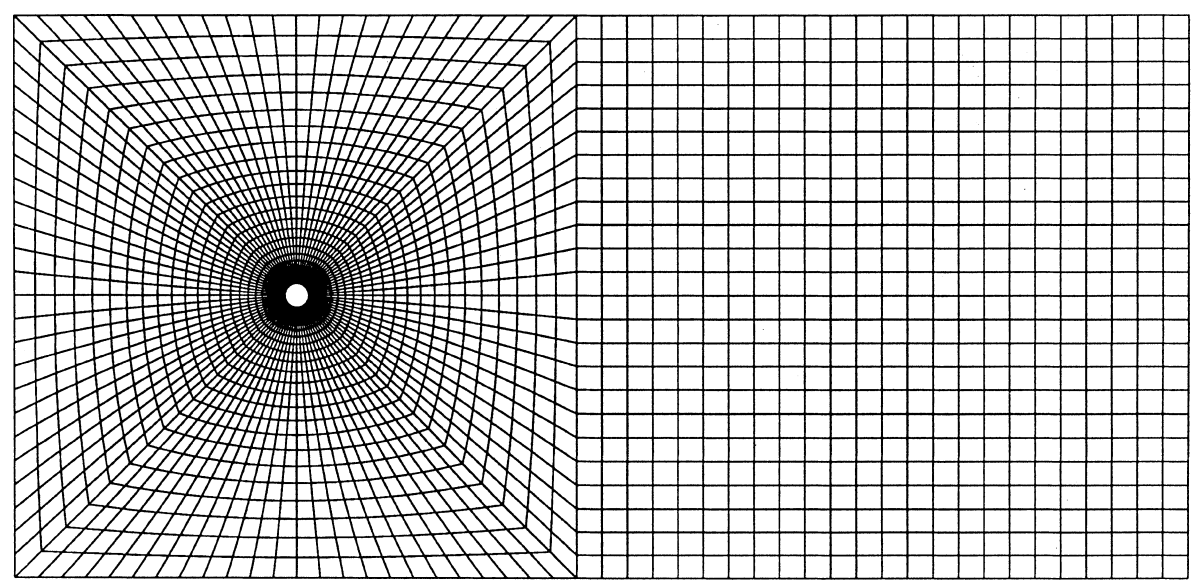

Fig. 13. Flow past a cylinder: mesh.

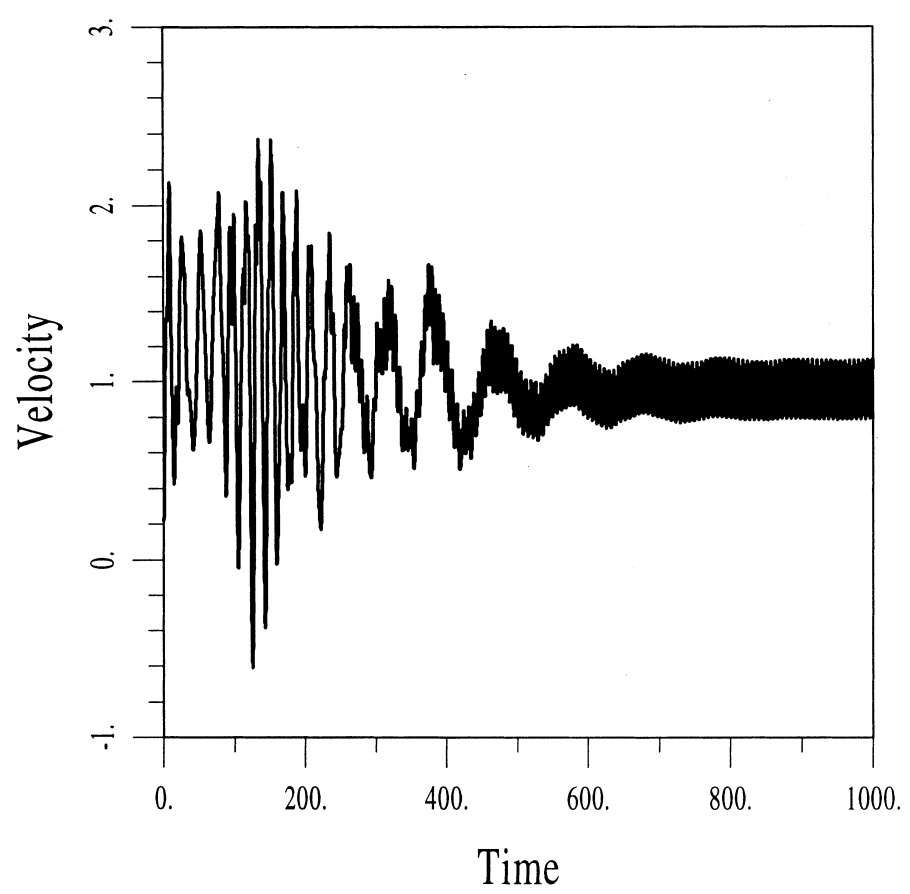

Fig. 14. Flow past a cylinder: nodal velocity history.

and thus other instabilities, such as that arising in convection dominated flows, have to be stabilized by other means.

Theoretical stability estimates and numerical examples demonstrate that the goal described is in fact achieved. The former can be obtained only with some weak assumptions on the finite element meshes employed and the velocity-pressure spaces. Numerical results show that the method is not only stable, but also that the formal accuracy of the time integration scheme is preserved by the use of the stabilization method.

From the computational point of view, the explicit treatment of the pressure gradient projection allows to adapt codes based on mixed div-stable formulations to equal order interpolations only with minor modifications. The continuity equation has to be modified by introducing a Laplacian-like term for the 


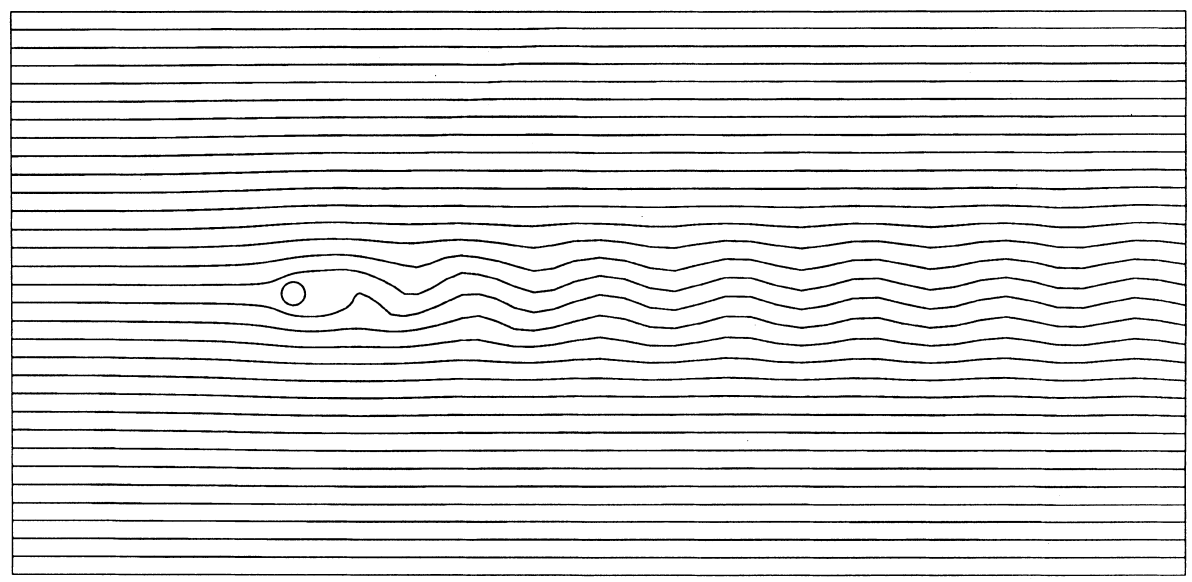

Fig. 15. Flow past a cylinder: streamlines.

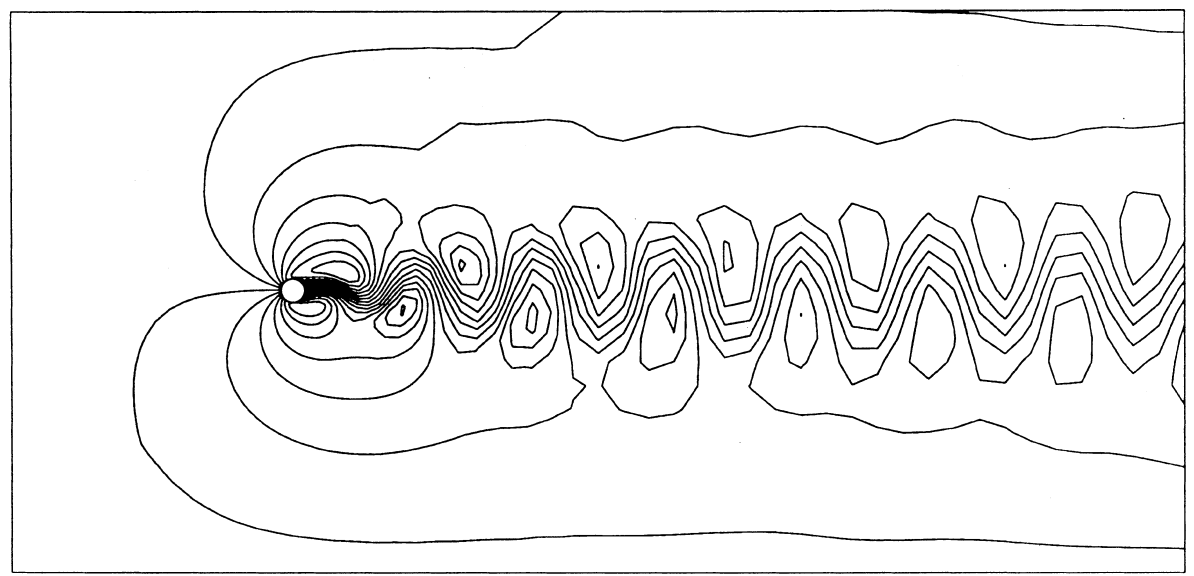

Fig. 16. Flow past a cylinder: stationary streamlines.

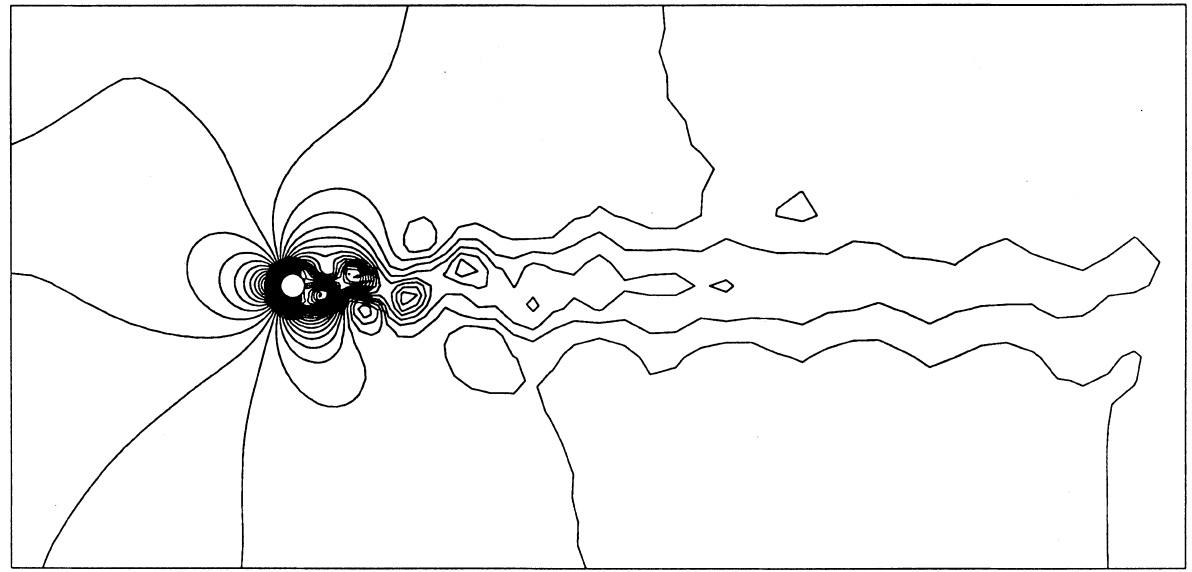

Fig. 17. Flow past a cylinder: pressure contours. 
pressure and the divergence of the pressure gradient projection in the RHS. We have shown how to apply the stabilization technique to both monolithic and fractional step time integration methods.

\section{References}

[1] R. Codina, J. Blasco, A finite element formulation for the Stokes problem allowing equal velocity-pressure interpolation, Comput. Meth. Appl. Mech. Engrg. 143 (1997) 373-391.

[2] R. Codina, J. Blasco, Analysis of a pressure stabilized finite element approximation of the stationary Navier-Stokes equations, Numerische Mathematik to appear.

[3] R. Codina, M. Vázquez, O.C. Zienkiewicz, A general algorithm for compressible and incompressible flow - Part III. The semi implicit form. Int. J. Numer. Meth. Fluids 27 1998, pp. 13-32.

[4] T.J.R. Hughes, L.P. Franca, M. Balestra, A new finite element formulation for computational fluid dynamics: V. Circumventing the Babuška-Brezzi condition: A stable Petrov-Galerkin formulation for the Stokes problem accommodating equal order interpolations, Comput. Methods Appil. Mech. Engrg, 59, 1986, pp. 85-99.

[5] F. Brezzi, J. Douglas, Stabilized mixed methods for the Stokes problem, Numerische Mathematik 53 (1988) $225-235$.

[6] L.P. Franca, S.L. Frey, Stabilized finite element methods: II. The incompressible Navier-Stokes equations, Comput. Methods Appl. Mech. Engrg. 99 (1992) 209-233.

[7] L. Tobiska, R. Verfürth, Analysis of a streamline diffusion finite element method for the Stokes and Navier-Stokes equations, SIAM J. Numer. Anal. 33 (1996) 107-127.

[8] R. Temam. Navier-Stokes Equations, North-Holland, Amsterdam, 1984.

[9] F. Brezzi, R. Falk, Stability of higher order Taylor-Hood elements, SIAM J. Numer. Anal. 28 (1991) 581-590.

[10] R. Stenberg, Error analysis of some finite element methods for the Stokes problem, Math. Comput. 54 (1990) $495-508$.

[11] R. Stenberg, A technique for analysing finite element methods for viscous incompressible flow, Int. J. Numer. Meth. Fluids 11 (1990) 935-948.

[12] J.G. Heywood, R. Rannacher, Finite element approximation of the non-stationary Navier-Stokes problem. IV: Error analysis for second order time discretization, SIAM J. Numer. Anal. 27 (1990) 353-384.

[13] A.J. Chorin, Numerical solution of the Navier-Stokes equations, Math. Comput. 22 (1968) 745-762.

[14] R. Temam, Sur l'approximation de la solution des équations de Navier-Stokes par la méthode des pas fractionaires (I), Arch. Rational Mech. Anal. 32 (1969) 135-153.

[15] P.M. Gresho, On the theory of semi-implicit projection methods for viscous incompressible flow and its implementation via a finite element method that also introduces a nearly consistent mass matrix Part I: Theory, Int. J. Numer. Meth. Fluids 11 (1990) $587-620$.

[16] G.E. Schneider, G.D. Raithby, M.M. Yovanovich, Finite element analysis of incompressible fluid flow incorporating equal order pressure and velocity interpolation, in: C. Taylor, K. Morgan, C.A. Brebbia (Eds.), Numer. Meth. Laminar and Turbulent Flow, Pentech Press, Plymouth, 1978.

[17] M. Kawahara, K. Ohmiya, Finite element analysis of density flow using the velocity correction method, Int. J. Numer. Meth. Fluids 5 (1985) 981-993.

[18] J.G. Rice, R.J. Schnipke, An equal order velocity-pressure formulation that does not exhibit spurious pressure modes, Comput. Methods Appl. Mech. Engrg. 58 (1986) 135-149.

[19] O.C. Zienkiewicz, R. Codina, A general algorithm for compressible and incompressible flow - Part I. The split characteristic based scheme, Int. J. Numer. Meth. Fluids 20 (1995) 869-885.

[20] R. Rannacher, On Chorin's projection method for incompressible Navier-Stokes equations, Volume Lecture Notes in Mathematics, 1530, Springer, Berlin, 1992, pp. 167-183.

[21] J.L. Guermond, L. Quartapelle, On the approximation of the unsteady Navier-Stokes equations by finite element projection methods. Numerische Mathematik 80 (1998) 207-238.

[22] A.J. Chorin, The numerical solution of the Navier-Stokes equations for an incompressible fluid. AEC Research and Development Report, NYO-1480-82. New York University, New York, 1967.

[23] C.E. Pearson, Report No. SRRC-RR-64-17, Sperry-Rand Research Center, Sudbury, Mass, 1964.

[24] J. Blasco, R. Codina, Space and time error estimates for a pressure stabilized, finite element method for the incompressible Navier-Stokes equations. Appl. Numer. Math., submitted.

[25] T.E. Tezduyar, J. Liou, D.K. Ganjoo, Incompressible flow computations based on the vorticity-stream function and velocitypressure formulations, Comput. Struc. 35 (1990) 445-472.

[26] M. Behr, J. Liou, R. Shih, T.E. Tezduyar, Vorticity-stream function formulation of unsteady incompressible flow past a cylinder: Sensitivity of the computed flow field to the location of outflow boundary, Int. J. Numer. Meth. Fluids 12 (1991) 323-342.

[27] M.A. Behr, D. Hastreiter, S. Mittal, T.E. Tezduyar, Incompressible flow past a circular cylinder: Dependence of the computed flow field on the location of the lateral boundaries, Comput. Methods Appl. Mech. Engrg. 123 (1995) 309-316.

[28] J.C. Simo, F. Armero, Unconditional stability and long term behavior of transient algorithms for the incompressible NavierStokes equations, Comput. Methods Appl. Mech. Engrg. 111 (1994) 111-154.

[29] T.E. Tezduyar, S. Mittal, S.E. Ray, R. Shih, Incompressible flow computations with stabilized bilinear and linear equal order interpolation velocity-pressure elements, Comput. Methods Appl. Mech. Engrg. 95 (1992) 221-242. 